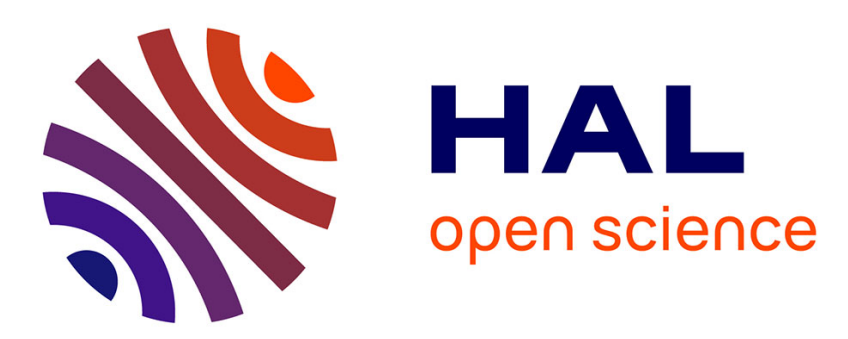

\title{
Numerical analysis of the linear and nonlinear vortex-sound interaction in a T-junction
}

Michaël Bauerheim, Edouard Boujo, Nicolas Noiray

\section{To cite this version:}

Michaël Bauerheim, Edouard Boujo, Nicolas Noiray. Numerical analysis of the linear and nonlinear vortex-sound interaction in a T-junction. AIAA AVIATION FORUM, Jun 2020, Virtual Event, United States. pp.1-23. hal-02923535

\section{HAL Id: hal-02923535 \\ https://hal.science/hal-02923535}

Submitted on 27 Aug 2020

HAL is a multi-disciplinary open access archive for the deposit and dissemination of scientific research documents, whether they are published or not. The documents may come from teaching and research institutions in France or abroad, or from public or private research centers.
L'archive ouverte pluridisciplinaire HAL, est destinée au dépôt et à la diffusion de documents scientifiques de niveau recherche, publiés ou non, émanant des établissements d'enseignement et de recherche français ou étrangers, des laboratoires publics ou privés. 


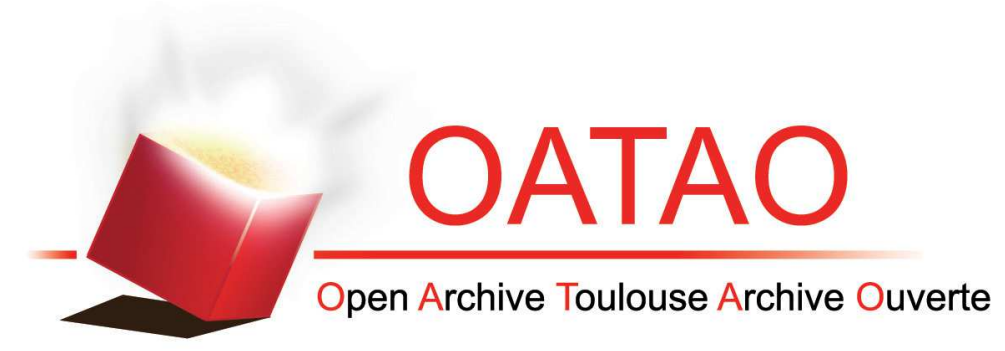

\section{Open Archive Toulouse Archive Ouverte (OATAO)}

OATAO is an open access repository that collects the work of some Toulouse researchers and makes it freely available over the web where possible.

This is an author's version published in: https://oatao.univ-toulouse.fr/26594

Official URL: https://doi.org/10.2514/6.2020-2569

\section{To cite this version :}

Bauerheim, Michaël and Boujo, Edouard and Noiray, Nicolas Numerical analysis of the linear and nonlinear vortexsound interaction in a T-junction. (2020) In: AIAA AVIATION FORUM, 15 June 2020 - 19 June 2020 (Virtual Event, United States).

Any correspondence concerning this service should be sent to the repository administrator: tech-oatao@listes-diff.inp-toulouse.fr 


\title{
Numerical analysis of the linear and nonlinear vortex-sound interaction in a T-junction
}

\author{
M. Bauerheim* \\ ISAE-Supaéro, Toulouse, 31400, France \\ E. Boujo ${ }^{\dagger}$ \\ Ecole Polytechnique, Palaiseau, 91128, France \\ N. Noiray \\ ETHZ, Zurich, 8092, Switzerland
}

\begin{abstract}
T-junctions correspond to a classical academic configuration employed to unravel the vortexsound interaction leading to self-sustained oscillations. It is composed of a closed deep cavity exposed to a low-Mach grazing flow, in which an unstable shear layer can develop. Many studies usually consider this hydrodynamic instability either as a "flapping shear layer", or as a "discrete vortex shedding", which then couples with the acoustic field. This paper follows the idea that these two descriptions are related to the linear and non-linear regimes of the shear layer response to acoustic waves, and thus intends to further analyze these regimes and their transition. To do so, a typical T-junction turbulent flow is computed by forced Large Eddy Simulation (LES) where acoustic waves are injected at several amplitudes. The flow response is extracted, and exhibits a linear regime as well as two distinct non-linear regimes where a partial saturation of the response occurs. The post-processing of the flow field in the three situations reveals that a flapping mechanism exists at low wave amplitudes, whereas a vortex shedding appears for highest acoustic levels. For moderate wave amplitudes, the behavior of the shear layer lies between these two classical views. This suggests that during self-sustained oscillations, a transition between these scenarios occurs, starting from a flapping motion followed by a vortex shedding.
\end{abstract}

\section{Introduction}

Inherent features of unsteady compressible turbulent flows are the generation of acoustic waves and vortices. Contrary to vorticity waves [1] which are convected by the mean flow at the speed $U_{b}$, acoustic perturbations have the unique property to propagate at a specific velocity known as the sound speed $c_{0}=\sqrt{\gamma P_{0} / \rho_{0}}$, where $P_{0}$ and $\rho_{0}$ are the mean pressure and density, and $\gamma$ is the ratio of the heat capacities. In subsonic flows $\left(U_{b}<c_{0}\right)$, it implies that such a perturbation can travel in the upstream direction, thus being able to interact with the very source responsible of the acoustic oscillations.

In non-reactive flows, acoustic waves can lock on coherent vortex shedding. When this interaction is destructive, efficient vortex-induced damping can be achieved [2, 3] by transferring acoustic energy to vortical modes. However, when a constructive coupling occurs, absolute instabilities [4] grow in the flow leading to undesirable effects like mechanical vibrations and tonal noise [5]. These instabilities are a nagging issue encountered in many practical applications containing connected pipes, or cavities with a grazing flow [6]-8]. A typical academic configuration is the T-junction where a side branch of length $L_{T}$ and width $W_{X}$ connects a main cavity. For deep cavities $\left(L_{T} \gg W_{X}\right)$, the acoustic mode in the side branch can excite the shear layer instability at the junction, resulting in vortex-driven self-sustained oscillations [9]. Such a configuration has been investigated for years using theory [5, 10, 11], experiments [12,-16] and more recently simulations [17,-19].

From these studies, the global mechanisms governing the interaction between acoustics and the unsteady shear layer in deep cavities with a low-Mach number grazing flow have been discovered and analyzed since the 70s. In particular, the vortex-sound theory established by Howe [20] and the linearized energy balance equation developed by Myers [21]

\footnotetext{
*Associate Professor, ISAE-Supaéro DAEP, michael.bauerheim@isae-supaero.fr

${ }^{\dagger}$ Assistant Professor, Ecole Polytechnique LadHyX, edouard.boujo@ladhyx.polytechnique.fr

†Professor, ETHZ CAPS, noirayn@ethz.ch
} 


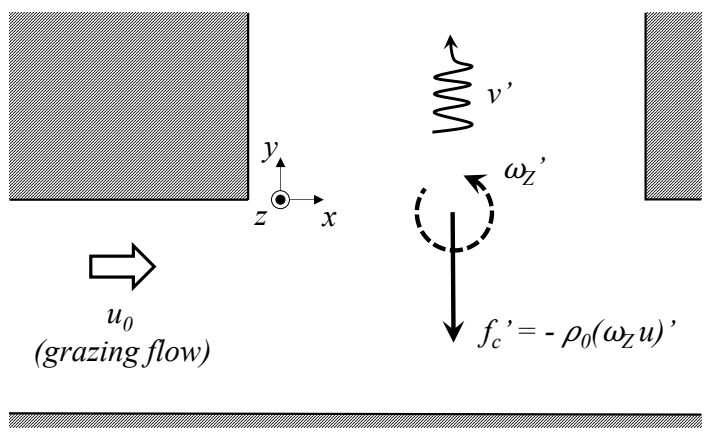

Fig. 1 Schematic of the vortex-sound interaction due to the unsteady Coriolis force $f_{c}^{\prime}=-\rho_{0} \omega_{Z}^{\prime} u_{0}<0$. It is assumed that vorticity is generated during the suction, i.e., when the transverse acoustic velocity is positive $v^{\prime}>0$. It leads a negative product $P=f_{c}^{\prime} v^{\prime}<0$ during the suction period. Then, during the blow-off $\left(v^{\prime}<0\right)$, the power becomes positive.

and Brear et al. [22] assert that acoustic energy can be produced when acoustic waves interact with a vortical flow because of the unsteady Coriolis force (figure 1$) f_{c}^{\prime}=-\rho_{0}(\vec{\omega} \times \vec{u})^{\prime}$, where $\rho_{0}$ is the mean density, $\vec{\omega}=\operatorname{curl}(\vec{u})$ is the vorticity, and $\vec{u}$ is the velocity field. When friction and compressible effects can be neglected, the time-averaged acoustic source power $\left\langle P_{\Omega}\right\rangle_{T}$ generated in the control volume $\Omega$ is given by [20]

$$
\left\langle P_{\Omega}\right\rangle_{T}=-\left\langle\int_{\Omega} \rho_{0}(\vec{\omega} \times \vec{u})^{\prime} \cdot \vec{u}^{\prime} d \Omega\right\rangle_{T},
$$

where $\langle.\rangle_{T}$ denotes the time-averaging over the period $T$. Usually, in this integral, only the contribution to the unsteady Coriolis force due to the unsteady vorticity $-\rho_{0}\left(\vec{\omega}^{\prime} \times \vec{u}_{0}\right)$ is retained for basic explaination of the vortex-sound coupling. Since the bulk velocity $\vec{u}_{0}$ is aligned with the main cavity grazing flow, equation (1) suggests that the Coriolis force is transverse to the flow direction (y-axis, figure 1 , and therefore that the acoustic power $\left\langle P_{\Omega}\right\rangle_{T}$ can be generated only by transverse acoustic waves. This examplifies why a T-junction is considered here as a canonical case to study the vortex-acoustics interaction. This theoretical background allows the understanding of the basic mechanisms responsible for the global response of the shear layer to acoustic waves. Based on equation (1), the key ingredient is the vorticity production at the junction. In a simplistic view, two main families of vorticity production can be distinguished: (1) the continuously oscillating or flapping shear layer [23-25] (figure 2-a), and (2) the so-called "discrete vortex model" [26-30] (figure 22-b) where the inward acoustic velocity (i.e. during the succion, $t=t_{1}$ in figure 2]) triggers a vortex shedding at the upstream edge.

These models have been studied extensively in the literature [25, 30, 31] and therefore these two scenarios constitute the baseline understanding of the vortex-sound theory. However, almost no studies consider that these two representations of the flow can apply for the same configuration: the flow is either described by a perturbed continuous vortex sheet (figure 2 -a), or by a single convected vortex (figure 2-b). Such a simplistic view has been recently renewed by Ma et al. [31] and reviewed by Morris [32]. They suggest that the actual vorticity field lies between these two scenarios. Specifically, Ma et al. [31] combines these two models since they observed that the upstream region ressembles a flapping vortex sheet, whereas further downstream the roll-up induces a detached vortex. Recently, Dai et al. [33] have shown, using vortex tracking simulations, that a flapping motion occurs at off-resonance conditions, whereas a concentrated vortex is created at the resonance state.

Following the idea of Dai et al. [33], the vortex sheet is affected by small perturbations during its initial growth, so that linearized theories and tools can be applied [34, 35]: this is known as the "linear regime", where the Coriolis force $f_{c}^{\prime}$ evolves linearly with the acoustic perturbation $v^{\prime}$, thus the acoustic source power is evolving as $v^{\prime 2}$. In such a case, the discrete vortex model can be questioned since no vortex is actually created, as observed in the vortex tracking simulations of Dai et al. [33]. However, after a substantial amplification of the acoustic mode in the cavity, the shear layer may experience large perturbations, which can modify its response to acoustic waves. This situation is known as the "non-linear regime", and is usually investigated thanks to the so-called describing-function introduced by Krylov and Bogoliubov [36] as an approximate procedure to analyse nonlinear control problems: the non-linear system is approximated by a linear time-invariant (LTI) transfer function depending on the amplitude $A$ of the system's input, here corresponding to the amplitude of the acoustic excitation [29, 30]. Recently, [15], [11], and [18] have investigated the 

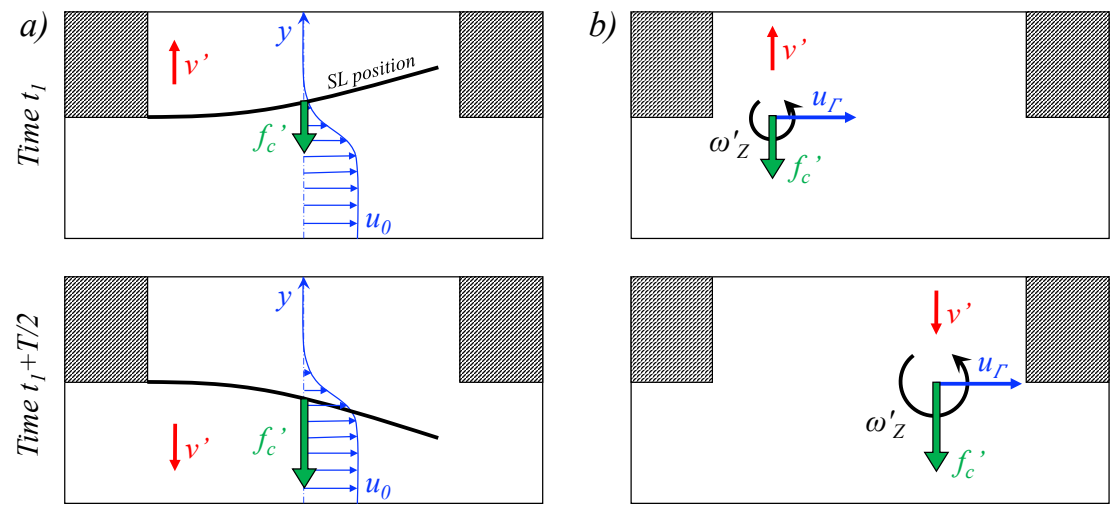

Fig. 2 Schematic view of the two scenarios: the flapping shear layer (a, left), and the discrete vortex (b, right). Two instants are displayed, corresponding to an opposite phase of the acoustic velocity $v^{\prime}$, i.e. $t_{2}=t_{1}+T / 2$.

response of a shear layer to several excitation amplitudes through describing functions, revealing its partial saturation for large perturbations, associated with large scale vortical structures. Nevertheless, no clear correlation between the linear/non-linear regimes and the underlying vorticity behavior has been drawn.

Consequently, this paper investigates numerically the acoustic power generation inside an academic T-junction in both the linear and nonlinear regimes. The objective is to identify the mechanisms responsible for the non-linearities, and to analyze how the flow evolves from one regime to another during the growth of self-excited oscillations. The case corresponds to a deep cavity of ratio $W_{X} / L_{T}=1 / 3$ which experiences a low-Mach number grazing flow ( $\left.M=U_{b} / c_{0} \approx 0.16\right)$. First in Section II the vortex-sound theory is introduced, and applied to the two phenomenological scenarios proposed in the literature: the flapping shear layer (figure 2-a), and the discrete vortex model (figure 2 -b). Then, the configuration of the T-junction and the numerical setup are described in Section III. An unforced simulation is computed, which reveals a self-sustained excited mode at $750 \mathrm{~Hz}$ due to the vortex-sound coupling. To control the wave amplitude and analyze the linear and non-linear regimes, the acoustic feedback loop is disconnected and acoustic waves are injected into the CFD domain at the specific frequency $750 \mathrm{~Hz}$ and several amplitudes $A / U_{b}$, from $0.05 \%$ up to $40 \%$. The describing function which relates the shear layer response to the input acoustic wave is extracted is Section IV. indicating that a saturation occurs for amplitudes $A / U_{b}$ larger than $0.4 \%$ : this is the first non-linear regime denoted $N_{1}$, where the gain of the describing function evolves as $A^{-0.5}$. Interestingly, a second non-linear regime is also observed, called $N_{2}$, with a distinct slope of the gain, varying like $A^{-0.3}$. In Section $\mathrm{V}$ the flow obtained at several amplitudes is analyzed and compared with the two scenarios established in Section II.C Results show that the flow continuously evolves between the two scenarios, starting from an oscillating shear layer at low amplitudes (linear regime), and transitioning to a vortex shedding at the upstream corner at higher acoustic levels (the second non-linear regime $N_{2}$ ). In the first non-linear regime $N_{1}$ corresponding to moderate acoustic levels, the flow exhibits both a flapping motion and an intense roll-up. These results show that the shear layer response to acoustic waves has a different spatio-temporal behavior during the initial growth of the mode and the final limit cycle, and thus that the transition between the flapping motion and the discrete vortex shedding is of crucial interest.

\section{Vortex-sound theory}

\section{A. Mathematical framework}

Vortices are a well-known feature of flows, in particular through turbulence, which still challenges research and computational physics. Turbulence can induce a broad-band noise [37], but vortices can also appear as coherent structures shed at specific times, as for example behind a cylinder with the so-called Von-Karman street. In such a case, a tonal noise might occur, and can be amplified if vortices couple with acoustics. Such a typical situation appears when a deep cavity (i.e. when the ratio of the diameter $W_{X}$ over the depth $L_{T}$ is smaller than one: $W_{X} / L_{T}<1$ ) is excited by a grazing flow. Consequently, this paper investigates the vortex-noise interaction inside a T-junction (Figure 3 ) where the grazing flow can become unstable, inducing a large vorticity intensity. Such an unstable flow acts as an acoustic 


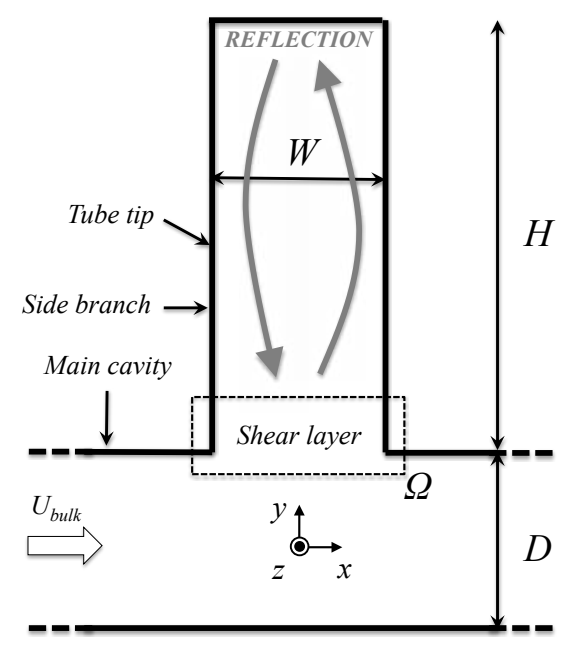

Fig. 3 Sketch of the T-junction where the unstable shear layer acts as an acoustic dipole generating acoustic waves. They propagate in the side branch and are reflected back (curved arrows) toward the vortex sheet.

dipole and generates acoustic waves which propagate in the side branch. They are reflected back toward the vortex sheet leading to a vortex-noise coupling. A key ingredient to understand this interaction is the response of the vortex sheet to the incoming acoustic wave.

While a monopolar source term is due to volume fluctuations as for an unsteady flame (non homogeneous mass balance equation), vorticity generates noise through a dipolar source term corresponding to the unsteady part of the Coriolis force $\vec{f}_{c}=-\rho \vec{\omega} \times \vec{u}[20]$, where $\vec{\omega}$ is the vorticity, i.e. the curl of the velocity field $\vec{u}$. Consequently, vortices act on the momentum balance equation. Starting from the Euler's equation in the Crocco's form [3] allows the introduction of the Coriolis force in the momentum equation. The linearization at first order of the momentum balance equation leads to

$$
\frac{\partial \vec{u}^{\prime}}{\partial t}+\vec{\nabla} B^{\prime}=\frac{\vec{f}_{c}^{\prime}}{\rho_{0}}
$$

where $\vec{f}_{c}{ }^{\prime}$ is the unsteady Coriolis force, for which only one term is usually retained: $\vec{f}_{c}^{\prime}=-\rho_{0}\left(\vec{\omega}^{\prime} \times \vec{u}_{0}\right)$ where $\rho_{0}$ and $\vec{u}_{0}$ are mean flow quantities, $\vec{\omega}^{\prime}$ is the unsteady vorticity, and $B^{\prime}=p^{\prime} / \rho_{0}+\vec{u}^{\prime} \cdot \vec{u}_{0}$, which is often approximated by $B^{\prime} \approx p^{\prime} / \rho_{0}$ at low Mach number. This balance equation is of fundamental interest for the vortex sound interaction since it is the starting point for both the acoustic jump conditions used in network modeling and to obtain the non-homogeneous Helmholtz equation. Taking the divergence of equation (2) and adding the time-derivative of the classical linearized mass equation yields the non-homogeneous wave equation

$$
\frac{1}{c_{0}^{2}} \frac{D_{0}^{2} B^{\prime}}{D t^{2}}-\nabla^{2} B^{\prime}=-\vec{\nabla} \cdot\left(\frac{f_{c}^{\prime}}{\rho_{0}}\right)
$$

where $D_{0} / D t$ is the convective time derivative. As for every problems involving hydrodynamic instabilities, the mean flow is a crucial ingredient for the Coriolis source term because of its convective nature $\left(u_{0}\right.$ appears explicitly in the linearized Coriolis force expression of the RHS of equation (3)), but can be neglected at first order for the acoustic propagation, so that $D_{0}^{2} / D t^{2} \approx \partial^{2} / \partial t^{2}$. Equation 3 is the classical non-homogeneous wave equation, showing that the unsteady Coriolis force $f_{c}^{\prime}$ acts as a dipole source/sink term for the sound generation.

In the wave equation (3), the generation of vorticity $f_{c}^{\prime}$ triggered by acoustics is not modeled. As proposed by Krylov and Bogoliubov [36], and introduced for the vortex-sound interaction by Kook and Mongeau [29] and [30], the response of the shear layer to acoustic waves can be characterized using a describing function $\mathcal{F}$, which relates the Fourier transform of $f_{c}^{\prime}$, denoted $\hat{f}_{c}$, to the transverse acoustic wave velocity $\hat{v}$ defined by its angular frequency $\omega$ and amplitude $A=|\hat{v}|:$

$$
\frac{\mathcal{F}(\omega, A, \vec{x})}{u_{0}}=\frac{\hat{f}_{c}(\omega, A, \vec{x})}{\hat{v}(\omega, A)}=G(\omega, A, \vec{x}) e^{j \Phi(\omega, A, \vec{x})},
$$


where $u_{0}$ is a normalization velocity, chosen here to be equal to the mean bulk velocity. The describing function is decomposed into a gain $G$, and a phase $\Phi$. Note that in the linear regime where $A$ is small, the function $\mathcal{F}$ does not depend on the amplitude $A$, and is therefore a transfer function with a constant gain: $G(\omega, A)=G(\omega)$. The gain $G$ and phase $\Phi$ can be evaluated numerically or experimentally by computing the unsteady Coriolis force, or any other similar quantity as the unsteady vorticity, the pressure jump over the vortex sheet [15], or the complete transfer matrix of the T-junction [16, 19]. Once $\mathcal{F}$ is known, the acoustic wave generation or the vortex-noise coupling can be analyzed in the linear and non-linear regime using equation 3 or through an energy balance equation.

\section{B. The energy point of view}

The vortex-noise coupling can affect the stability of the configuration, leading to the exponential growth of acoustic oscillations in the side branch during the linear regime, followed by a saturation due to non-linear mechanisms. The response of the shear layer to acoustic waves is one of the most important source of non-linearities in the system, and its study is the main objective of this paper. This non-linearity is revealed by a gain and a phase of the describing function $\mathcal{F}$ depending on the amplitude $A$ of the oscillation. In particular, a decrease of the gain is expected, and has been quantitatively observed by Graf and Ziada [15] and Nakiboglu et al. [18]. The stability of the system excited by the unsteady Coriolis force can be studied using the energy balance equation, which can be recast as

$$
\frac{\partial E_{\Omega}}{\partial t}=P_{\Omega}-I_{\partial \Omega}
$$

using the zero Mach number assumption, where $E_{\Omega}$ is the acoustic energy integrated over the domain $\Omega$ encapsulating the shear layer, $I_{\partial \Omega}$ is the acoustic flux at the boundaries, and $P_{\Omega}$ corresponds to a production or damping term. For the vortex-sound interaction, the energy source term can be expressed as $P_{\Omega}=\int_{\Omega} \vec{f}_{c}^{\prime} \cdot \vec{u}^{\prime} d \Omega$. In particular, it implies that no interaction occurs between vortices and acoustics when the vortex sheet is located at a velocity node $\left(\vec{u}^{\prime}=\overrightarrow{0}\right)$. The same reasoning concludes that vortex-noise is of crucial importance when the vortex sheet is located at a velocity anti-node, as it is in quarter-wave resonators such as the present T-junction. The application of a time-averaging $\langle\cdot\rangle_{T}$ on the energy balance equation (5) indicates that the system becomes unstable if the vortex-sound coupling exceeds the losses, i.e., $\left\langle P_{\Omega}\right\rangle_{T}-\left\langle I_{\partial \Omega}\right\rangle_{T}>0$. In such a case, the oscillations grow exponentially: this is the linear regime where $\left\langle P_{\Omega}\right\rangle_{T}$ scales as $A^{2}$. At larger amplitudes, a non-linear regime occurs where the Coriolis force, and thus the power source term, saturates. When the production and loss terms compensate each other, the system oscillates at a constant amplitude, known as the limit cycle.

In the following, several definitions of the power source term will be computed to highlight its spatial or time-varying behavior. From the local power source term due to the Coriolis force $P_{X Y T}(x, y, t)=f_{c}^{\prime}(x, y, t) v^{\prime}(x, y, t)$, several integrated power quantities can be derived, defined as

$$
\begin{array}{r}
P_{X T}(x, t)=\frac{1}{L_{y}} \int_{y} f_{c}^{\prime}(x, y, t) v^{\prime}(x, y, t) d y, \\
P_{X Y}(x, y)=\left\langle f_{c}^{\prime}(x, y, t) v^{\prime}(x, y, t)\right\rangle_{T}, \\
P_{X}(x)=\frac{1}{L_{Y}} \int_{y}\left\langle f_{c}^{\prime}(x, y, t) v^{\prime}(x, y, t)\right\rangle_{T} d y, \\
P_{T}(t)=\frac{1}{W_{X} L_{Y}} \iint_{x, y} f_{c}^{\prime}(x, y, t) v^{\prime}(x, y, t) d x d y .
\end{array}
$$

These definitions highlight space and time correlations between the response of the shear layer (generation of unsteady vorticity) and the acoustic field. Note that the acoustic velocity is not reduced to its time component only, because the junction cannot be assumed compact, especially in the non-linear regime, as detailed in Section ??. In the following, these definitions of the power source term will be used to analyze the forced LES flows, which requires first to characterize a typical flapping shear layer and vortex shedding thanks to $P_{X T}$ and $P_{X Y}$.

\section{Application to simplified models of a flapping motion and vortex shedding}

To fix the ideas, and to highlight key differences between the two scenarios proposed in figure 2 in terms of power source terms, 2D phenomenological models are introduced for the flapping motion and the discret vortex models. More realistic models are available in the literature [23,-25, 28,-30], nevertheless here only simplified phenomenological models will be applied for the sake of simplicity. 

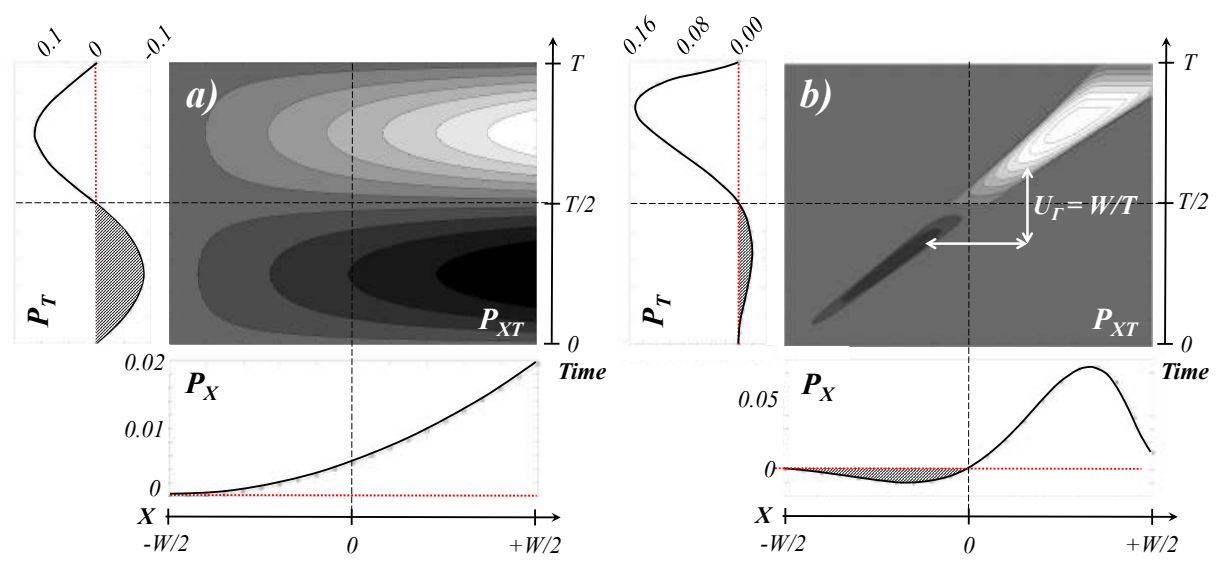

Fig. 4 The y-integrated power source term $P_{X, T}$ is displayed for the two phenomenological models: the flapping shear layer (left, a), and the discrete vortex (right, b). White zones correspond to a production of sound, whereas dark is associated with damping regions. The time-integrated $\left(P_{T}\right.$, left) and spatially integrated $\left(P_{X}\right.$, bottom) power sources are also displayed.

(i) The flapping shear layer: this model corresponds to a standing oscillation of the shear layer, as shown in figure 2 -a. Howe [24] and Chatelier et al. [25] proposed semi-analytical approaches to evaluate the shape of the shear layer, and its motion in time. Here, a simplistic phenomenological model is adopted, where the vorticity $\omega_{Z}(x, y, t)$ is set to 1 for $h_{-}(x, t)<y<h_{+}(x, t)$, and 0 otherwise, where $h_{ \pm}(x, t)$ is defined as:

$$
h_{ \pm}(x, t)=\left(x+\frac{W}{2}\right) \frac{H}{W}\left[\sin \left(\frac{2 \pi t}{T}\right) \pm 1\right]
$$

For the parameters $H / W=2 \%$ and a normalized baseline flow $u_{0} / U_{b}=1-\tanh (2 y / H)$, the power source terms $P_{X T}, P_{X}$ and $P_{T}$ are displayed in figure 4 a. $P_{X T}$ reveals the standing pattern of the oscillations where the whole shear layer oscillates in phase. Since the shear layer moves inward the cavity when the acoustic velocity points inward $\left(v^{\prime}>0\right)$, it leads to a damping term for the first half of the period $(0<t<T / 2)$, whereas a constructive coupling occurs for $T / 2<t<T$. Thus, the net production/damping of sound is controlled by the asymmetry between the upward and downward motion of the shear layer. A perfectly symmetric case would lead to $P_{X}=0$. Here the asymmetry is induced by the mean flow $u_{0}$ which gives weight to the outward motion $(y<0)$. In real applications, asymmetries may also arise from a non-symmetric motion and/or from a non-symmetric acoustic velocity (see figure 9 for an example). Since the shear layer is attached at the upstream corner, no unsteadyness is observed for $x$ close to $-W / 2$, leading to a null power as shown by $P_{X}(-W / 2)=0$. However, since the amplitude of the motion increases along the longitudinal coordinate, maximal power is obtained at the downstream edge.

(ii) The discrete vortex: the unsteady vorticity is driven by a vortex shedding synchronized with the acoustic velocity at the junction. For the sake of simplicity, the vortex is considered here as a perfect disk of radius $R_{v}^{0}$ shed at the location $x_{v}^{0}=-W / 2$ with a constant vorticity $\omega_{Z}(x, y, t)=1$. The vortex is then convected downstream at the bulk speed $U_{\Gamma}=U_{b} / 2=W / T$ (first hydrodynamic mode). Along its convection, the vortex grows exponentially, i.e. $R_{v}(t)=R_{v}^{0} e^{\sigma t}$. Results for $R_{v}^{0} / W=2 \%$ are shown in figure 4 b. The spatiotemporal power source term $P_{X T}$ indicates a "convected" oscillation, with a convective speed $U_{\Gamma} \approx W / T$. The vortex $\left(\omega_{Z}^{\prime}>0\right)$ is created when the acoustic velocity points inward the cavity $\left(v^{\prime}>0\right)$, leading to a damping term. Then, the vortical region is convected downstream. For $t>T / 2$, the acoustic velocity now points outward the cavity $\left(v^{\prime}<0\right)$, which generates noise at the vortex location. As shown by $P_{X}$, the net sound production is controlled by the vortex growth: if $\sigma=0$, then the damping and production term compensate each other, leading to a zero net production.

The typical power source results shown in figure 4 for the flapping motion and the vortex models exemplify that a flapping and a vortex shedding lead to different spatio-temporal behaviors of the shear layer and vortex-noise coupling. Consequently, such analysis will be applied to the LES results in order to identify the mechanism leading to the vortex-sound interaction in the T-junction. In particular, both $P_{X T}$ and $P_{X}$ allows a clear identification of the mechanism involved. For instance, in the discrete vortex model, a damping zone can be observed for $0<t<T / 2$ in $P_{X}$ because of the vortex generation, which is not the case for the flapping motion. This difference can be also observed using the 


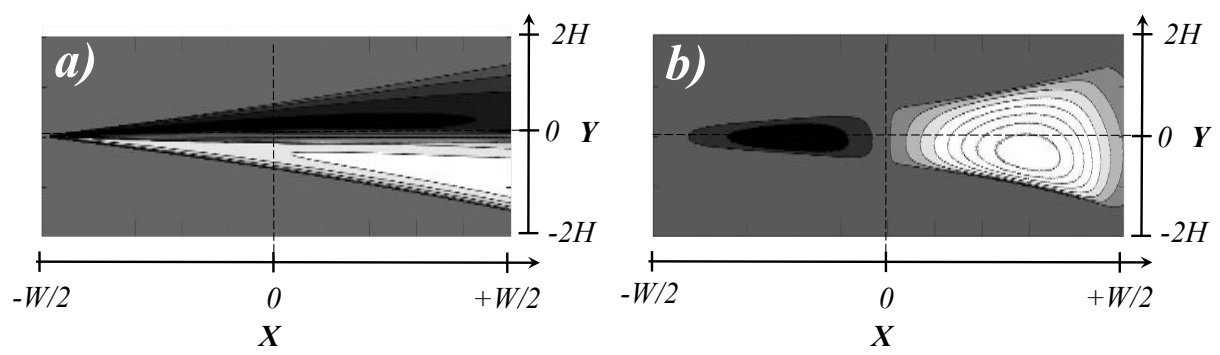

Fig. $5 P_{X Y}$ for the two scenarios: the flapping motion (a, left), and the vortex shedding (b, right). White zones correspond to a production of sound, whereas dark is associated with damping regions

spatial time-averaged source power $P_{X Y}$ (figure 5), revealing again a clear difference between the two scenarios. For the flapping shear layer, the damping and production zones are distributed along the y-direction, i.e. along the flapping direction. In contrast, theses zones are distributed along the x-direction for the discrete vortex model because of the vortex convection. Note that a combination of these two scenarios may arise, in particular when the flapping motion induces a downstream roll-up.

\section{Deep cavity whistling}

\section{A. The configuration and numerical setup}

To investigate the response of the vortex sheet to acoustic waves, a T-junction with $L_{T}=90 \mathrm{~mm}$ (figures 3 and 6 ) is investigated thanks to Large Eddy Simulation (LES). LES are performed using the code AVBP. It solves the filtered 3D compressible Navier-Stokes equations on unstructured meshes:

$$
\begin{aligned}
\frac{\partial \bar{\rho}}{\partial t}+\frac{\partial}{\partial x_{i}}\left(\bar{\rho} \tilde{u}_{i}\right) & =0 \\
\frac{\partial \bar{\rho} \tilde{u}_{i}}{\partial t}+\frac{\partial}{\partial x_{j}}\left(\bar{\rho} \tilde{u}_{i} \tilde{u}_{j}\right) & =-\frac{\partial}{\partial x_{j}}\left(\bar{P} \delta_{i j}-\bar{\tau}_{i j}-\bar{\tau}_{i j}^{t}\right), \\
\frac{\partial \bar{\rho} \tilde{E}}{\partial t}+\frac{\partial}{\partial x_{j}}\left(\bar{\rho} \tilde{E} \tilde{u}_{j}\right) & =-\frac{\partial}{\partial x_{j}}\left[\overline{u_{i}\left(P \delta_{i j}-\tau_{i j}\right)}+\bar{q}_{j}+\bar{q}_{j}^{t}\right]
\end{aligned}
$$

where $\bar{\rho}$ is the filtered density, $\tilde{u}_{i}$ is the $\mathrm{i}^{\text {th }}$-component of the velocity, $P$ is the static pressure, $\bar{\tau}_{i j}$ and $\bar{\tau}_{i j}^{t}$ are the resolved and turbulent viscous tensor, $\tilde{E}$ is the energy and $\bar{q}_{i}=-\bar{\lambda} \frac{\partial \tilde{T}}{\partial x_{i}}$ is the $\mathrm{i}^{\text {th }}$-component of the energy flux. The turbulent energy flux $\bar{q}_{i}^{t}=-\lambda^{t} \frac{\partial \tilde{T}}{\partial x_{i}}$ is evaluated by assuming a constant Prandtl number $P_{r}^{t}=0.6$.

At the junction, the typical cell size is $300 \mu m$, leading to $y^{+}<5$ at walls. Walls are therefore treated as no-slip and adiabatic. The model Sigma developped by Nicoud et al. [39] is adopted to account for the small turbulent scales: $\bar{\tau}_{i j}^{t}$ and $\bar{q}_{i}^{t}$. A turbulent mean velocity profile is injected at the inlet, characterized by a bulk velocity $U_{b}=56 m / s$ and a power-law profile where the exponent is set to $1 / 7$. Note however that no synthetic turbulence is injected at the inlet. The associated Reynolds number is $R_{e}=U_{b} W_{X} / v \approx 1.510^{5}$, where $v=1.1210^{-5} \mathrm{~m}^{2} \mathrm{~s}^{-1}$ is the kinematic viscosity of the air. Knowing that the width-to-depth ratio of the side cavity is $W_{X} / L_{T}=1 / 3$, interactions between the shear layer instability and the quarter-wave mode of the side tube are expected [9]. A numerical scheme developed by Colin and Rudgyard [40] with a third-order in space and forth-order in time, called TTG4A, is used for robustness and accuracy. The maximum cell size is $\delta x=1 \mathrm{~mm}$ which allows acoustic waves to be resolved on the grid $(N=10$ points per wavelength) for frequencies up to $f_{\text {max }}=c_{0} / N \delta x=34 \mathrm{kHz}$. For hydrodynamic modes which are convected at a lower speed, typically $0.4 U_{b}$ in the vortex sheet characterized by $\delta x=0.3 \mathrm{~mm}$, the maximal resolved frequency is $f_{\text {max }}=0.4 U_{b} / N \delta x=7466 \mathrm{~Hz}$. The upstream/downstream non-reflecting boundary conditions are imposed using the NSCBC formalism developed by ? ].

The boundary at the tube tip (figure 60 can be set as non-reflecting [41] to obtain a stable simulation (case $A$ ). From this case, the tube tip boundary condition is then imposed as perfectly reflecting to investigate potential unstable modes (case $B$ ). As shown in Section III.B, an unstable mode at $750 \mathrm{~Hz}$ grows exponentially in time until a limit cycle governed 


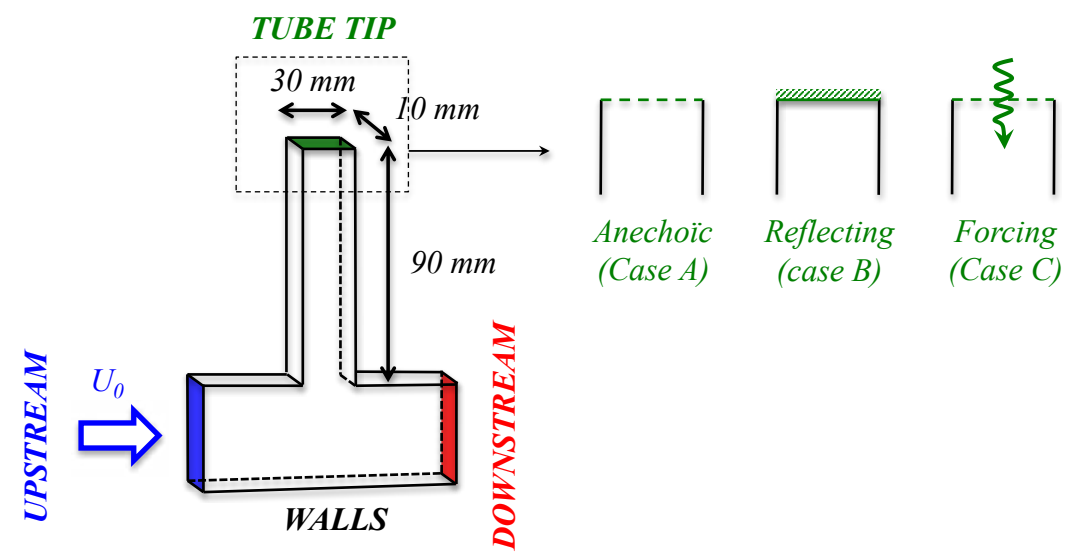

Fig. 6 Sketch of the T-junction used for LES. The length of the side tube is set to $L_{T}=90 \mathrm{~mm}$. Inlet and outlet boundaries conditions are non-reflecting [? ]. At the tube tip, the imposed condition can be switched between non-reflecting, reflecting, or being forced by external acoustic waves.

by a non-linear saturation. Since the only difference between case $A$ and $B$ is the acoustic treatment of the side tube, it ensures that the unstable mode is controlled by the vortex-sound coupling in the side branch. Finally, to further investigate the linear and non-linear regimes in order to identify their governing mechanisms (flapping motion or vortex shedding), forced simulations (Case $C$ ) are computed. They consist in simulations where a non-reflecting boundary condition is imposed at the tube-tip to let acoustic waves leaving the domain, while external acoustic waves are injected through the same boundary at a well-controlled frequency $f=750 \mathrm{~Hz}$ and amplitude $A / U_{b}$ from $0.04 \%$ to $50 \%$.

\section{B. Self-excited oscillations}

First, all the three boundary conditions are set to non-reflecting using a relaxed NSCBC approach [41] to ensure the non-reflection of acoustic waves for frequencies higher than $f_{c}=100 \mathrm{~Hz}$. In such a case, the system is stable (Case $A,---$ in figure 7), where only small pressure fluctuations at $1000 \mathrm{~Hz}$ are observed. The Strouhal number is $S_{t}=f W_{X} / U_{b}=0.53$, typical of Strouhal numbers where the second hydrodynamic mode is dominant (range $[0.5,0.7]$ ), as shown in figure 7] [18, 27]. This case is considered as stable.

Based on case $A$, the boundary condition is now switched to non-reflecting (case $B$ ). A strong coupling occurs, leading to pressure oscillations at $750 \mathrm{~Hz}$ and amplitude $\pm 250 \mathrm{~Pa}$ in the limit cycle. It corresponds to a self-excited mode where the frequency is associated with the quarter-wave mode of the tube. The Strouhal number is $S_{t}=0.40$, typical of instabilities involving the first hydrodynamic mode, as shown by the modal decomposition displayed in figure 7 (bottom right). It shows that the acoustic mode is unstable, potentially driven by the vorticity production at the junction. The oscillations grow exponentially in the linear regime (--- in figure 7), before reaching a plateau controlled by non-linearities where the source term balances acoustic losses. The growth rate is estimated at $22 \mathrm{~Hz}$, compared with the $750 \mathrm{~Hz}$ of the frequency of oscillations. The main objective of this paper is to investigate the transition from the linear (exponential growth) to the nonlinear regime (plateau). Since during the self-sustained oscillations the amplitude, but also the frequency, are not well-controlled, the saturation mechanism is investigated in the following by forcing the system with acoustic waves at $750 \mathrm{~Hz}$ and several amplitudes (case $C$ ). The vortex-sound coupling will be studied using the describing function introduced in equation (4), and a spatio-temporal analysis based on the power source terms developed in equations (6)-(9).

\section{Global non-linear response}

To investigate the vortex-driven mechanism yielding the instability observed in case $B$ (Section [III.B), the tube tip boundary condition is again set to non-reflecting (case $C$ ) to disconnect the acoustic feedback loop. In addition, acoustic waves are injected at a specific amplitude $A$ and a fixed frequency to focus on the $750 \mathrm{~Hz}$ mode. This approach is therefore quasi-static, since the behavior of case $B$ where the wave amplitude is changing in time is studied by 

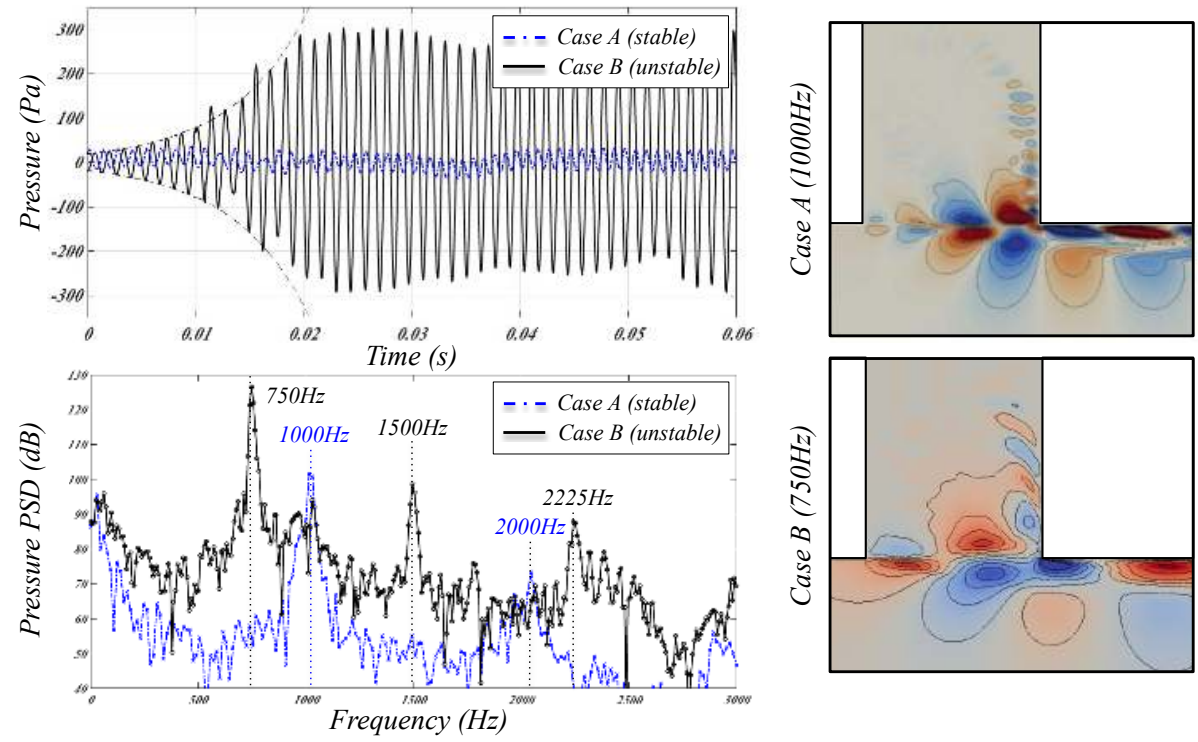

Fig. 7 Time-traces (top left) and PSD (bottom left) of the pressure at the tube tip for a non-reflecting (case $A$, -.. ), and a reflecting (case $B$, - ) boundary condition. The exponential growth for case $B$ is displayed by --- . The modal decomposition of case $A$ (top right, at $1000 \mathrm{~Hz}$ ) and $B$ (bottom right at $750 \mathrm{~Hz}$ ) are also shown.

investigating the flow field at several but independent constant forcing level $A$, each amplitude being computed separately. Additional simulations are performed at $800 \mathrm{~Hz}$ to analyze the results dependency with the forcing frequency. First, qualitative results on the global modal response of the shear layer will be drawn. Then, key ingredients to compute the describing function will be analyzed quantitatively. Finally, the global describing function of the shear layer will be obtained, in order to identify the linear and non-linear regimes.

\section{A. The modal response}

Results of the forced LES simulations (case $C$ ) are shown in figure 8 , which displays the Fourier transform at $750 \mathrm{~Hz}$ of the axial velocity fluctuations, denoted $\hat{u}$, for three different amplitudes: $A / U_{b}=0.22 \%$ (left), $A / U_{b}=1.78 \%$ (middle), and $A / U_{b}=8.92 \%$ (right). For all amplitudes ranging from $0.04 \%$ to $50 \%$, the mode at $750 \mathrm{~Hz}$ corresponds to the first hydrodynamic mode of the shear layer. Note that for amplitudes between $0.04 \%$ (not shown) and $0.4 \%$, the modal decompositions of the dominant mode at $750 \mathrm{~Hz}$ are very similar, showing that the underlying hydrodynamic mechanisms are equivalent. For higher amplitudes, spatial differences can be noticed between each case. To ease the comparison, the norm of the modal decomposition of the velocity at the frequency $f=750 \mathrm{~Hz}$ is displayed (bottom) along the $\mathrm{x}$-axis. It indicates that for small perturbations $\left(A / U_{b}=0.22 \%\right)$, the velocity perturbation $\hat{u}$ is constantly growing along the longitudinal direction, until the upstream edge at $x=W / 2$ where a singularity occurs. This singularity apart, the maximum of the velocity norm is highlighted by a white circle (o), located at $85 \%$ of the cavity for $A / U_{b}=0.22 \%$. Smaller vortices also exist in the recirculation zone close to the downstream edge.

It can be clearly observed that the hydrodynamic mode $\hat{u}$ is distorted for higher amplitudes $A / U_{b}>0.4 \%$. In particular, the location of the maximum is displaced further upstream, at $60 \%$ of the cavity width for $A / U_{b}=1.78 \%$, and close to the upstream edge ( $15 \%$ of the cavity width) for $A / U b=8.92 \%$. For amplitudes higher than $8.92 \%$, a similar hydrodynamic mode with a maximum close to the upstream corner is obtained. This first result suggests that a different mechanism is acting at low and large perturbations, with a dominant activity at the downstream edge at small acoustic levels, whereas it is located closer to the upstream corner for large perturbations. The case at $A / U_{b}=1.78 \%$ can be considered as an intermediate stage, where both the upstream and downstream contributions are relevant. This situation is the one encountered for the self-excited mode in case B, as shown by figure 7 (bottom right) where a similar modal decomposition is obtained.

Additionally, the forced simulations at $750 \mathrm{~Hz}$ and amplitudes $A / U_{b}$ are also analyzed at the frequency $f=1000 \mathrm{~Hz}$ (mode observed in the stable case $A,---$ in figure 8 ), and $f=1500 \mathrm{~Hz}$ (second hydrodynamic mode, $\cdots \cdots \cdots$ in figure 8 ). 


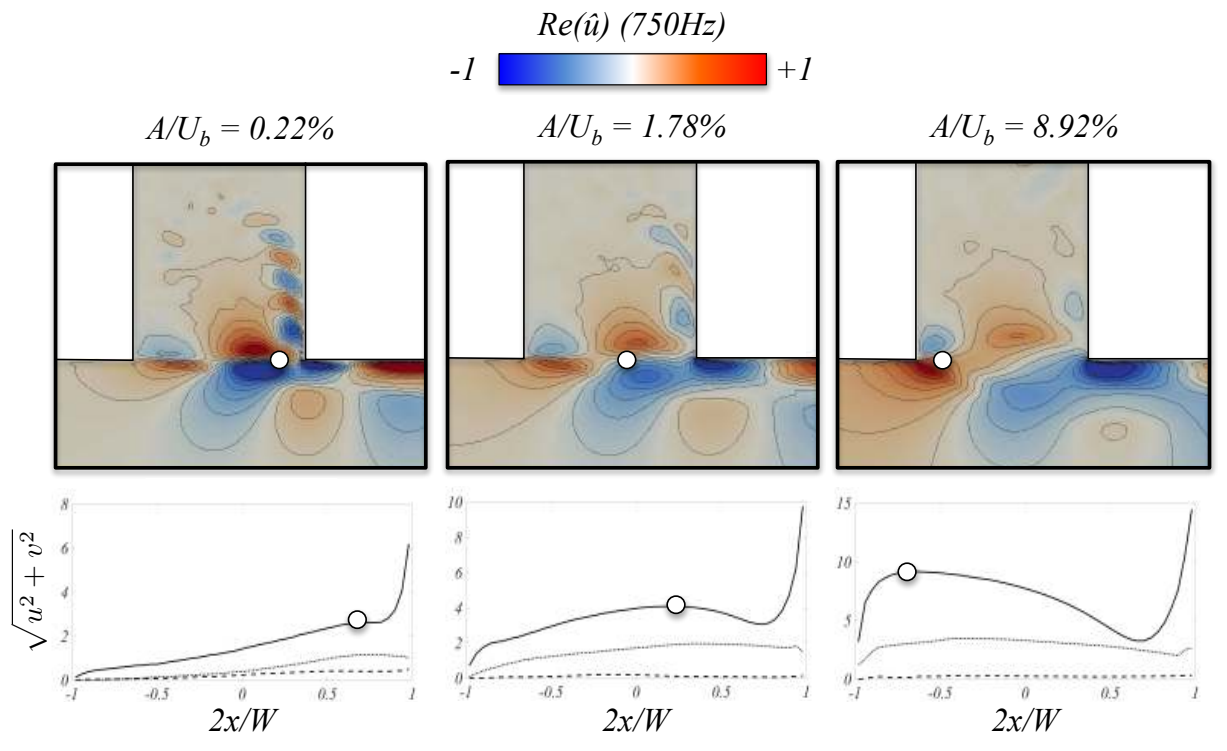

Fig. 8 Real part of the modal decomposition at $750 \mathrm{~Hz}$ (top), denoted $R e(\hat{u})$, of the normalized axial fluctuating velocity for three forced simulations: $A / U_{b}=0.22 \%$ (left), $A / U_{b}=1.78 \%$ (middle), and $A / U_{b}=8.92 \%$ (right). The Fourier coefficients at three frequencies $f$ of the velocity fluctuation are displayed (bottom) for each forcing amplitude $A / U_{b}: f=750 \mathrm{~Hz}(-), f=1000 \mathrm{~Hz}(---)$, and $f=1500 \mathrm{~Hz}(\cdots \cdots \cdots)$.

It shows that the mode at $1000 \mathrm{~Hz}$ obtained in case $A$ is not observed in the forced simulations, which is consistent with the simulation of case $B$ (figure 7). Moreover, the first harmonic at $1500 \mathrm{~Hz}$ is low but not negligible, especially at large amplitude forcing. Nevertheless, Boujo et al. [42] have shown that this harmonic is not a significant source of the modal dynamics at $750 \mathrm{~Hz}$ for the present configuration. It implies that the effect of harmonics can be discarded in the response of the shear layer, which is a prerequisite for using the concept of describing functions to analyze the non-linear regime: it can be assumed that the limit cycle is mainly governed by the saturation of the dominant mode at $750 \mathrm{~Hz}$, the non-linear generation of harmonics being less significant. In the following, the describing function proposed in equation (4) will be extracted from the LES, in order to quantitatively identify the transition between the linear and non-linear regime.

\section{B. The global describing function $\mathcal{F}_{g}$}

The describing function in equation (4) relates the Fourier transform of the Coriolis force $\hat{f}_{c}$ to the transverse acoustic forcing $\hat{v}$. This concept was first introduced to characterize the response of a shear layer to acoustic waves by Kook and Mongeau [29] and [30]. Recently, it has been reused by Graf and Ziada [15] and Nakiboglu et al. [18]. It allows an approximate description of the non-linear behavior of the shear layer, which is valid if the non-linear regime is controlled by the saturation of the dominant mode (harmonics having then a minor role in the non-linear response). This quantity is also relevant since the the power source $\left\langle P_{\Omega}\right\rangle_{T}$ in the energy balance equation, given in (5), can be recast using this particular function:

$$
\left\langle P_{\Omega}\right\rangle_{T}=\frac{1}{2} \operatorname{Re}\left(\int_{\Omega} \hat{f}_{c} \cdot \hat{v}^{*} d \Omega\right)=\frac{A^{2}}{2 u_{0}} \operatorname{Re}\left(\int_{\Omega} \mathcal{F} d \Omega\right)=\frac{A^{2}}{2 u_{0}} G_{g} \cos \left(\Phi_{g}\right)
$$

where $*$ denotes the complex transpose, and $\hat{v}$ is assumed constant in the domain $\Omega$ (compact assumption). Consequently, the global describing function

$$
\mathcal{F}_{g}(A, \omega)=\int_{\Omega} \mathcal{F} d \Omega=\frac{u_{0}}{\hat{v}} \int_{\Omega} \hat{f}_{c} d \Omega=G_{g}(A, \omega) e^{j \Phi_{g}(A, \omega)}
$$

is of key interest in the following, since associated with the global source power due to the vortex-sound coupling. As a reminder, in the linear regime $G_{g} \approx c s t$ so that $\left\langle P_{\Omega}\right\rangle_{T} \propto A^{2}$. The non-linearities can be quantified by the number $m$ where $\left\langle P_{\Omega}\right\rangle_{T} \propto A^{2-m}$, a complete saturation being characterized by $m=1$, and the linear regime by $m=0$. 


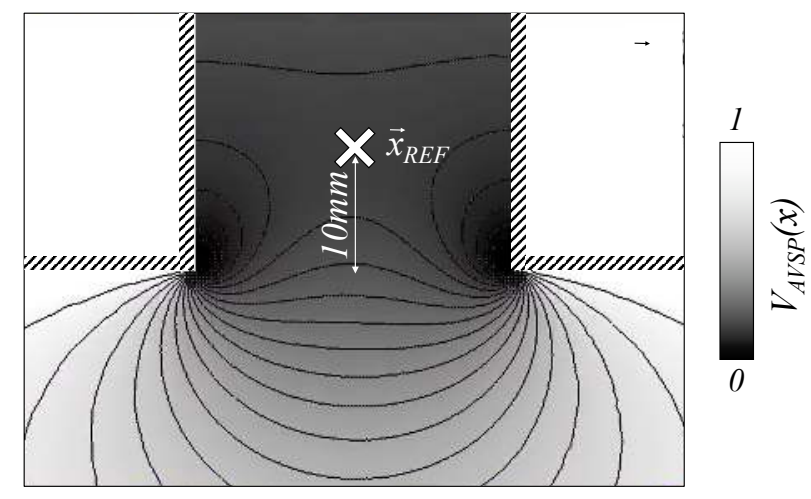

Fig. 9 Modulus of the $y$-component of the velocity mode at $750 \mathrm{~Hz}$ obtained by AVSP, called $V_{\mathrm{AVSP}}(\vec{x})$. This field is used to reconstruct the acoustic velocity fluctuations locally in the sub-domain $\Omega$, based on the time-varying signal extracted at the reference point $(\times)$. At the reference point, the velocity fluctuations are assumed to have a pure acoustic nature.

To extract this quantity by avoiding a complex triple decomposition in the LES to segregate acoustic and hydrodynamic velocity fluctuations, the transverse acoustic velocity $v^{\prime}$ along the $y$-direction is extracted at a reference point $\vec{x}_{r e f}$, close to, but outside, the shear layer. At this reference location, no hydrodynamic fluctuations are present and the velocity perturbation can therefore be considered as pure acoustics. To avoid assuming a uniform transverse acoustic velocity field $v^{\prime}(t, \vec{x})=v_{r e f}^{\prime}(t)$, a methodology based on an Helmholtz solver is considered to reconstruct the local acoustic velocity $v^{\prime}(t, \vec{x})$ based on this single reference point data $v_{r e f}^{\prime}(t)$. To do so, the acoustic field is reconstructed using a 3D Helmholtz solver [43] assuming a zero Mach number. The Helmholtz solution is shown in figure 9, displaying also the reference point located $10 \mathrm{~mm}$ above the shear layer. The data at the reference point is used to scale the acoustic field both in magnitude and phase. Thus, the acoustic transverse velocity is approximated by

$$
v^{\prime}(t, \vec{x})=v_{r e f}^{\prime}(t)\left|V_{A V S P}\right|(\vec{x})
$$

where $\left|V_{A V S P}\right|(\vec{x})$ is the modulus of the $y$-component of the velocity mode obtained by the AVSP code. Note that the phase of the velocity mode $\arg \left(V_{A V S P}\right)$ is not used, which assumes that the sub-domain $\Omega$ encapsulating the shear layer is compact regarding the acoustic wavelength. The validity of this compact assumption is discussed below, because depending on the choice of the sub-domain $\Omega$ used to extract the unsteady Coriolis force. Note also that no particular treatment have been applied at corners in the Helmholtz simulation, leading to singular points at these locations.

Several techniques can be employed to extract the unsteady Coriolis force from the LES simulations. The natural first question to address in the present framework is the choice of the subdomain $\Omega$ used in equation 15 . Figure 10 -a displays a numerical instantaneous schlieren view when the configuration is forced at $750 \mathrm{~Hz}$ and $A / U_{b}=1.78 \%$ (moderate amplitude oscillations). It highlights different vortical zones localized close to the T-junction, featuring a main vortex sheet (denoted MV, ---·) and side vortices (denoted SV, Consequently, a subdomain $\Omega_{m v}$ and $\Omega_{s v}$ can be defined to assess the role of these two vortex sheets in the global response, obtained by the subdomain $\Omega_{t o t}(---)$. Note that a proper subdomain $\Omega$ should satisfy two main constrains:

(i) Capturing the global Coriolis force of the system, otherwise part of the driven or damping phenomena will be missing.

(ii) Ensuring the compactness assumption regarding the acoustic wavelength considered, i.e. the subdomain size in the $\vec{y}$ direction, denoted $\|\Omega\|_{y}$, should be small compared with $\lambda$, i.e., $\|\Omega\|_{y}<<\lambda$. If this criterion is not valid, classical analytical modeling assuming a compact vortex sheet would fail, as well as the equation (16) used to reconstruct the acoustic field inside $\Omega$ because of the constant phase assumption.

Note that for the whole domain $\Omega_{t o t}$ as well as for $\Omega_{s v}$, this compact assumption is not valid, because for example $\left\|\Omega_{t o t}\right\|_{y} \approx \lambda / 4$ which is not small compared with $\lambda$. However, this assumption holds for $\Omega_{m v}$, but a question remains: "How relevant is the subdomain $\Omega_{m v}$ ?", since side vortices (not taken into account in $\Omega_{m v}$ ) could potentially affect the total response of the T-junction. Figure 10 b shows that such side vortices play only a minor role. Indeed, the axial velocity $u_{0}$ is low in $\Omega_{s v}$, thus the Coriolis force $\vec{f}_{c}^{\prime} \cdot \vec{y} \approx-\rho_{0} \omega_{Z}^{\prime} u_{0}$ remains low despite the high level of vorticity induced by the side vortices. Consequently, the subdomain $\Omega_{m v}$ is chosen: it contains the main source term of noise 

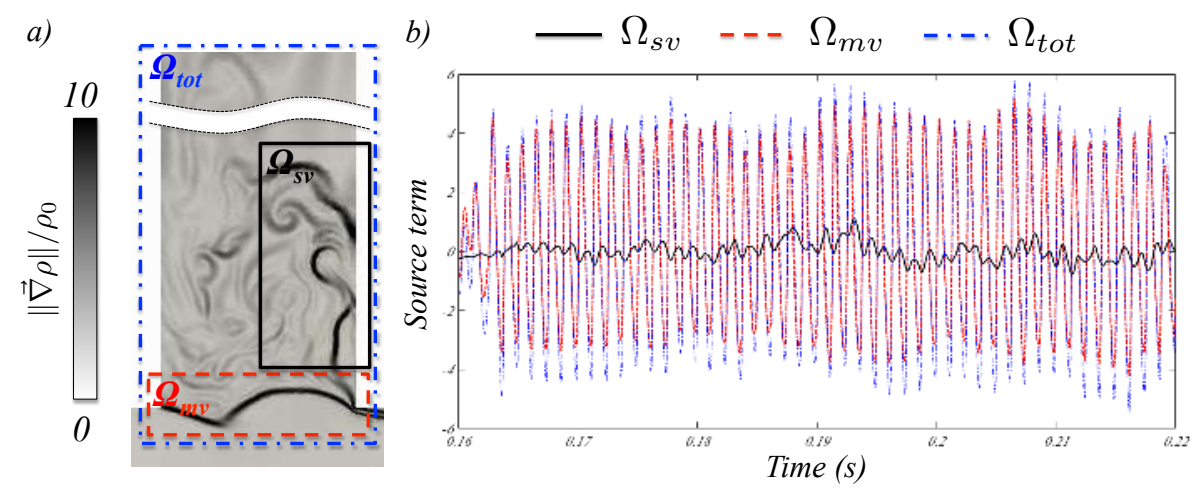

Fig. 10 a) Instantaneous numerical Schlieren view in case $C$ at $750 \mathrm{~Hz}$ and $A / U_{b}=1.72 \%$, highlighting two vortical zones: the main vortex sheet (MV, ---· ) at the junction, and side vortices (SV, — ) induced by the main vortex sheet impinging the right corner. b) Source term $\int_{\Omega}\left(\vec{f}_{c}^{\prime} \cdot \vec{y}\right) d \Omega$ obtained for three subdomains: $\Omega_{m v}$, $\Omega_{s v}$ and the whole T-junction domain $\Omega_{t o t}$.

while remaining compact regarding the acoustic wavelength. Note that the describing function is in this study based on the Coriolis force $f_{c}^{\prime}$, and not on the classical approximation $f_{c}^{\prime} \approx-\rho_{0} \omega_{Z}^{\prime} u_{0}$ or on the pressure jump across the shear layer as done by Graf and Ziada [15]. This ensures that the complete response of the shear layer is taken into account, especially at large acoustic levels. Further details are provided in Appendix A

The normalized gain $G_{g} / u_{0}$ of the global describing function (equation $(15)$ ) is displayed in figure 11 for the forcing frequencies $f=750 \mathrm{~Hz}$. LES results obtained by a forcing at $800 \mathrm{~Hz}$ ( $\mathbf{a})$ are also indicated, highlighting the sensitivity of the describing function with the forcing frequency. In particular, results at $f=800 \mathrm{~Hz}$ show large discrepancies at low wave amplitude, mainly because of the sharp peak in the linear regime. This justifies the forcing method employed to ensure a well-controlled frequency and amplitude of the forcing, instead of analyzing the self-excited mode obtained in case $B$. Further details are provided by Boujo et al. [42] where the gain of the describing function is obtained for various amplitudes and frequencies using a linearized approach. Note that in figure 11 , the value of $A / U_{b}$ corresponds to the measured value of the transverse acoustic velocity at the reference point, which therefore might differ slightly from the expected forcing amplitude.

Based on figure 11, three main regimes can be observed:

(i) A linear regime ( $L$ in figure 11 for low amplitudes, here $A / U_{b}<0.4 \%$, characterized by a constant gain. Indeed, in such a regime, the describing function reduces to the transfert function of a LTI (Linear Time-invariant) system with $G_{g}(A, \omega)=G(\omega)$.

(ii) A non-linear regime $\left(N_{1}\right.$ in figure 11 where the gain evolves with the amplitude $A / U_{b}$. As in Nakiboglu et al. [18], the gain in the non-linear regime varies approximately like $1 / \sqrt{A}$. A question remains on how universal is this square root power low characterizing the first non-linear regime. Note that the complete saturation of the shear layer (i.e., the unsteady Coriolis force $f_{c}^{\prime}$ is constant with the amplitude $A$ ) would lead to $G_{g}(A, \omega) \propto 1 / A$. It suggests that in the present non-linear regime, the shear layer is still responding to the acoustic waves, but another mechanism is lowering its amplitude. This regime corresponds to the one obtained at the limit cycle of case B.

(iii) A third regime $\left(N_{2}\right.$ in figure 11$)$ is also observed. It corresponds to a non-linear regime where the gain varies like $A^{-0.3}$ instead of $A^{-0.5}$.

The global describing function has revealed three different regimes, one linear $(L)$ and two non-linear $\left(N_{1}\right.$ and $\left.N_{2}\right)$. The transition between the linear regime $(L)$ and the first non-linear regime $\left(N_{1}\right)$ occurs at $A / U_{b} \approx 0.4 \%$, so that the left case of figure 8 is representative of the linear regime, and the middle case corresponds to the first non-linear regime. The transition between the regime $N_{1}$ and $N_{2}$ occurs at $A / U_{b} \approx 10 \%$, thus this situation corresponds to the case $A / U_{b}=8.92 \%$ shown in figure 8 (right). A similar behavior with two distinct non-linear regimes where also found in the literature [15], yet no particular details were provided on this phenomenon. In Nakiboglu et al. [18], the forcing amplitude is limited to $A / U_{b}<10 \%$, which prevents the second non-linear regime $\left(N_{2}\right)$ to be observed.

The combination of the modal decomposition (figure 8) and the describing function (figure 11) provides an overview of the behavior of the shear layer in presence of transverse acoustic waves. In the linear regime, all modal decompositions are similar (therefore only $A / U_{b}=0.22 \%$ is displayed), showing a high level of unsteadiness at the downstream edge (o in figure 8). The transition between the linear and non-linear regime occurs when the location of this high level of 


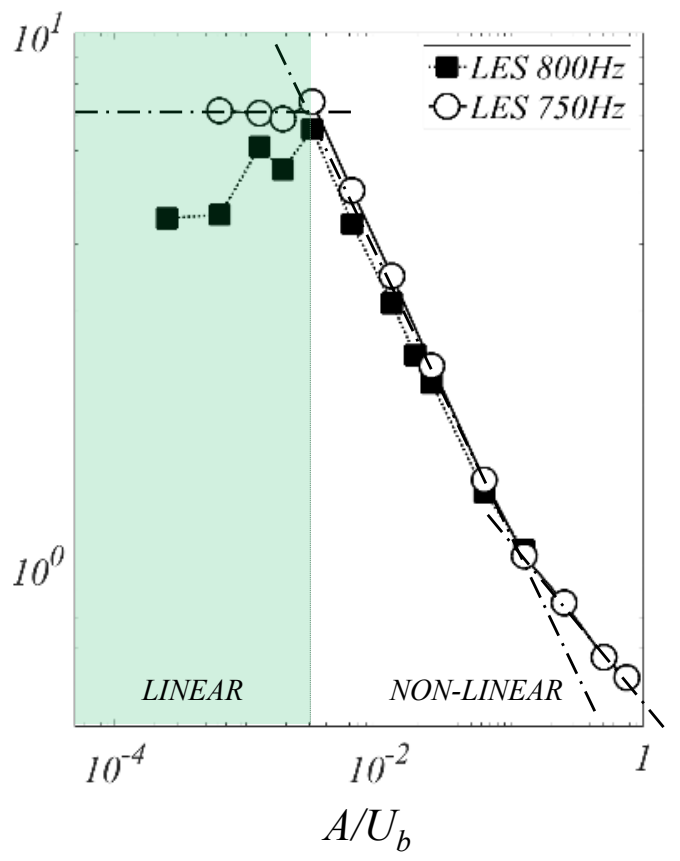

Fig. 11 Normalized gain of the global describing function $G_{g} / u_{0}$ (equation $(15)$ ) vs. the wave amplitude $A / U_{b}$, for case $C$ with two forcing frequencies: $f=750 \mathrm{~Hz}(\circ)$, and $f=800 \mathrm{~Hz}(\boldsymbol{\square})$. Three regimes are also identified $\left(L, N_{1}\right.$, and $\left.N_{2}\right)$.

unsteadiness moves further upstream, typically $50 \%$ of the cavity width for $A / U_{b}=1.78 \%$. The last transition between the two non-linear regimes $\left(N_{1}\right.$ to $\left.N_{2}\right)$ is characterized by the maximal unsteadiness attached to the upstream corner ( $15 \%$ of the cavity width for $\left.A / U_{b}=8.92 \%\right)$. For higher amplitudes $\left(A / U_{b}>8.92 \%\right)$, this unsteadiness cannot moves further upstream. To study these transitions in details, a further analysis is required to understand the spatio-temporal behavior of the shear layer motion.

\section{Spatio-temporal analysis}

\section{A. From the flapping motion to the vortex shedding}

To understand the differences between the three regimes, an in-depth look at the flow is required. First, instantaneous snapshots of the forced LES simulations at amplitudes $A / U_{b}=0.22 \%$ (linear regime $L$ ), $A / U_{b}=1.72 \%$ (first non-linear regime $N_{1}$ ), $A / U_{b}=8.92 \%$ (second non-linear regime $N_{2}$ ), and $A / U_{b}=35.7 \%$ are displayed in figure 12 . At low amplitudes (top), corresponding to the linear regime, the shear layer exhibits a flapping motion, leading downstream to a weak roll-up because of the shear layer interacting with the downstream edge. At moderate acoustic levels $\left(A / U_{b}=1.72 \%\right)$, the roll-up appears further upstream, in this particular case at approximately $50 \%$ of the cavity width. At this intermediate stage, corresponding to the first non-linear regime in figure 11, the roll-up is intense, yet a single detached vortex is not actually created. The flapping leaves the place to a pure vortex shedding at the upstream corner for larger amplitudes, typically $A / U_{b}=8.92 \%$. Such a vortex is created at the upstream corner when the acoustic velocity points inward the side-tube (succion), which constitutes the starting point of numerous analytical models developed to study the vortex-sound interaction. This is also the main feature characterizing the second non-linear regime $N_{2}$. Note that the radius of the vortex increases with the wave amplitude, as shown between $A / U_{b}=8.92 \%$ and $A / U_{b}=35.7 \%$ : even at these large wave amplitudes, the vorticity field is still changing drastically with the amplitude $A$, which may explain that the complete saturation of the gain, i.e. $G_{g}(A, \omega) \propto 1 / A$, is never reached (figure 11 ). A deviation of the vortex away from the side-tube is also observed, which limits the vortex-corner interaction at the downstream edge. 


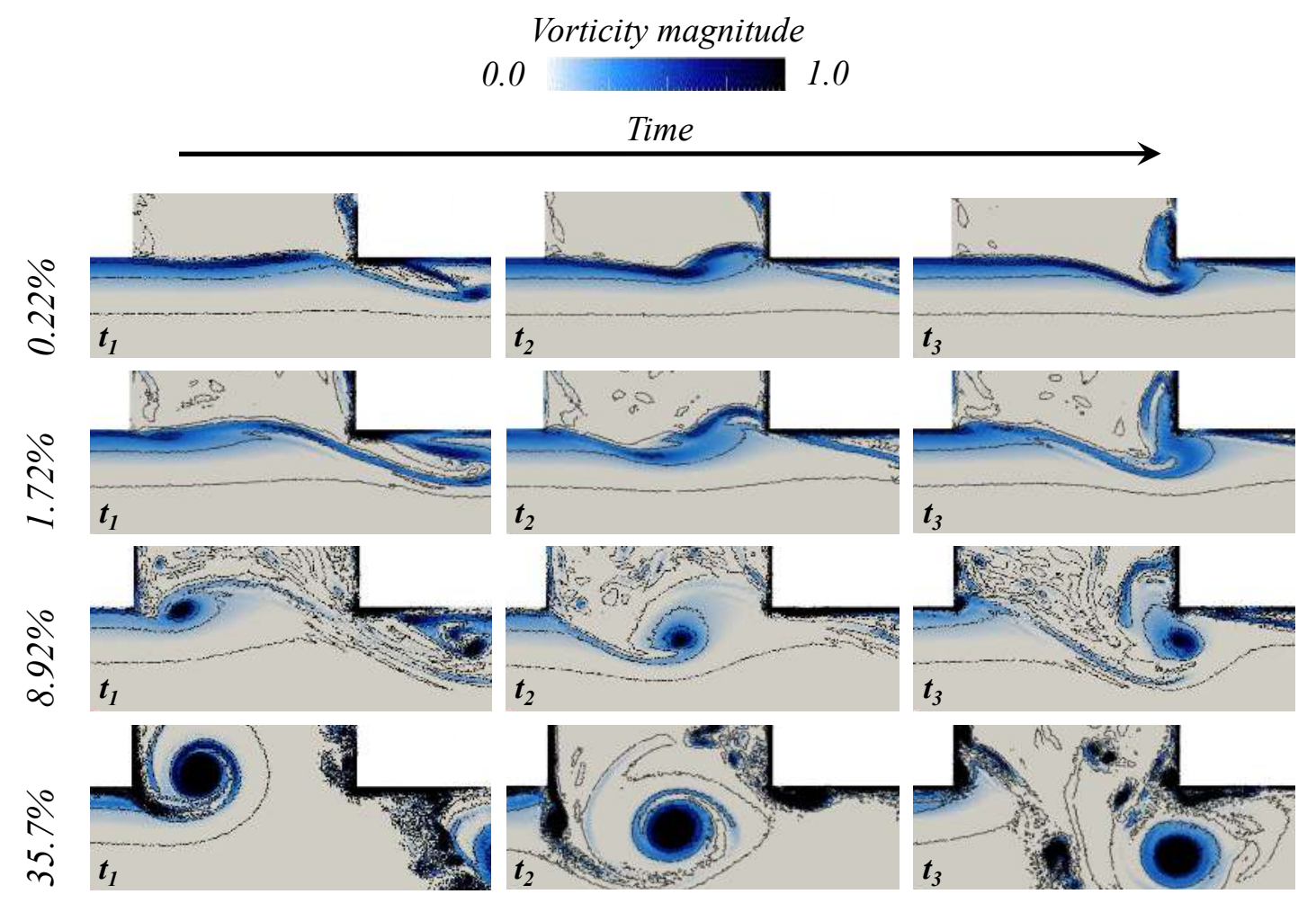

Fig. 12 Vorticity magnitude in case $\mathbf{C}$ at three different instants $\left(t_{1}\right.$ to $\left.t_{3}\right)$ for various forcing amplitudes: $A / U_{b}=0.22 \%, A / U_{b}=1.78 \%, A / U_{b}=8.92 \%$, and $A / U_{b}=35.7 \%$.

Moreover, at the highest amplitudes $\left(A / U_{b}>15 \%\right)$, a second discrete vortex is generated at the upstream corner, when the acoustic velocity points outward the side-tube, i.e. during the blow-off, because of the motion of the upstream boundary layer. This second vortex is still shed at a frequency close to $750 \mathrm{~Hz}$, but in opposite rotation compared with the first main vortex. Since $\omega_{Z}^{\prime}$ and $v^{\prime}$ are both negative (for the main vortex, they were both positive), it yields a negative power source during this second vortex creation (as for the main vortex, since $P_{X Y T}=-\rho_{0} \omega_{Z}^{\prime} u_{0} v^{\prime}<0$ ). It implies that the creation of a vortex at the upstream edge, either the first or second one, always absorbs a part of the acoustic energy. This intuitive result was also observed using the phenomenological model established in Section III.C, where a damping zone lies close to the upstream corner for the discrete vortex model (figure 5 -b). Note that in comparison, the flapping motion generates both a damping and production zones at the upstream corner (figure 5 -a).

\section{B. Space-time correlation of the vortex-sound coupling}

The temporal snapshots have indicated that the transitions between the three regimes $\left(L, N_{1}\right.$, and $\left.N_{2}\right)$ might be controlled by the location of the roll-up and the vortex creation. To further explore these transitions and understand the fundamental differences between the three regimes, a space-time correlation of the power source term $P_{X T}$ is computed. For the post-processing, a correlation between the unsteady Coriolis force $f_{c}^{\prime}$ and the acoustic velocity $v^{\prime}$ is required, which implies the knowledge of the acoustic field in the sub-domain $\Omega_{m v}$ : as discussed in Section IV.B, this step is performed using the acoustic solution $V_{A V S P}$ of the problem computed by the Helmholtz solver AVSP. Based on the LES simulations, the Coriolis force is extracted following the procedure detailed in Section IV.B A phase-averaging operator is applied on the filtered Coriolis force and velocity perturbations to avoid spurious oscillations due to turbulence fluctuations, the mode at $1000 \mathrm{~Hz}$ at low amplitudes, as well as higher harmonics at large forcing amplitudes. Results are given in figure 13

In the linear regime $\left(A / U_{b}=0.22 \%\right)$, a standing pattern similar to figure 4 a can be observed. In the $x-t$ plane, it is characterized by damping and production zones distributed along the vertical axis, typical of a flapping motion. It highlights that close to the upstream corner, no fluctuation exists because the shear layer is attached. However, the amplitude of the oscillations increases further downstream, to be maximum close to the downstream edge. 


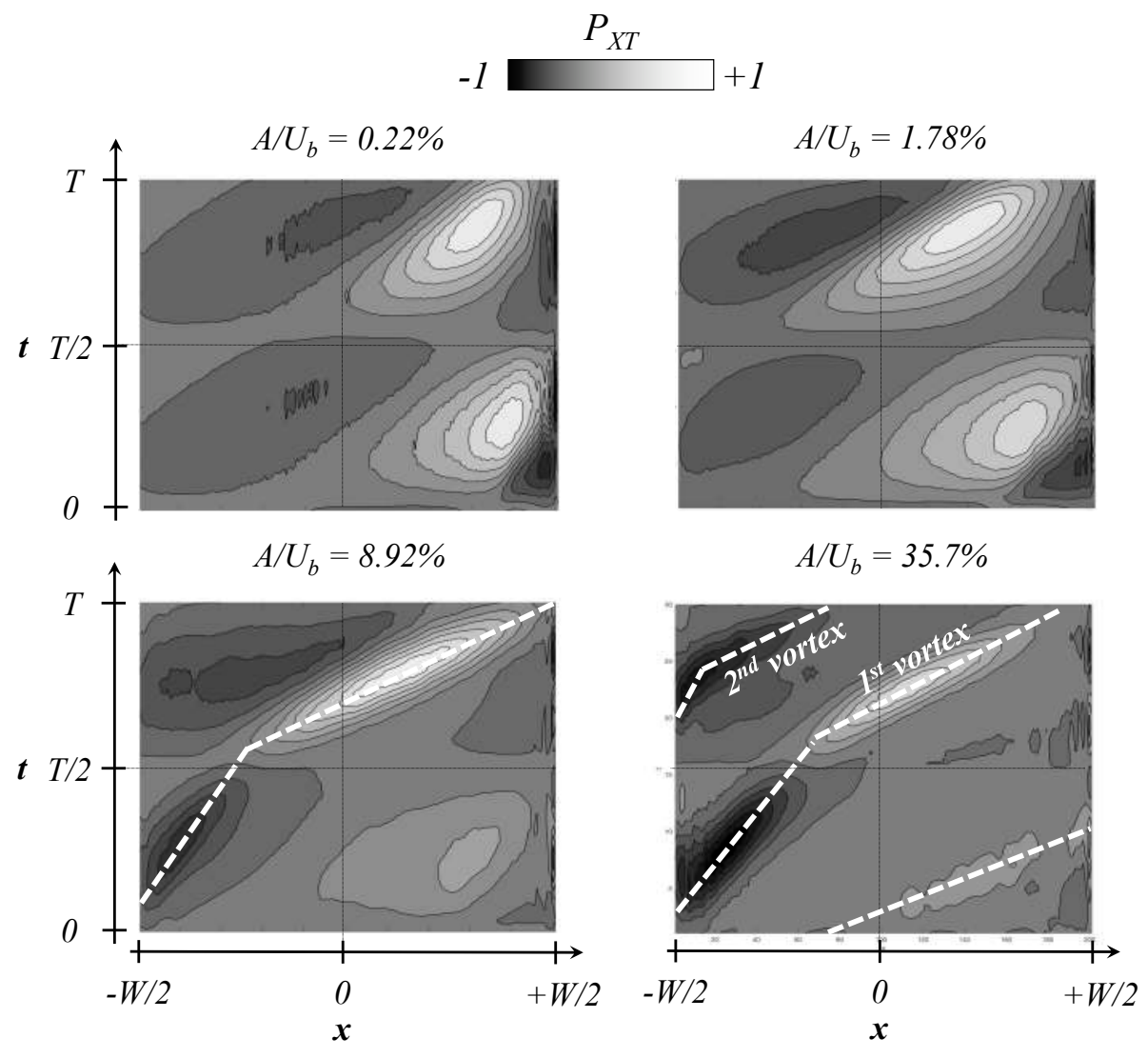

Fig. 13 Phase-averaged power source $P_{X T}$ at $750 \mathrm{~Hz}$ for several amplitudes $A / U_{b}$. Results are adimensionalized, white zones corresponding to a positive power, and black to negative values. The dash lines highlight a convective oscillation of speed $u_{\Gamma}=d x / d t$.

In the first non-linear regime $\left(N_{1}, A / U_{b}=1.78 \%\right)$, such a standing pattern is still visible for $0<x<W / 2$. Nevertheless, another weak damping and production zones appear further upstream $(-W / 2<x<0)$. It reveals that in this regime, the shear layer is oscillating closer to the upstream corner, as suggested by the modal decomposition in figure 8 The roll-up in this case cannot be clearly observed because the space-time correlation is still dominated by the flapping motion. However, it can be noticed that now the damping/production zones are also aligned along the line $x / W=t / T$, which shows the convective nature of some of the oscillations at the speed $U_{\Gamma}=W / T$ (dashed line in figure 13). Consequently, this regime exhibits a combination of a standing flapping motion and a weak convective oscillation, and is therefore an intermediate stage between the fully flapping motion of the linear regime $(L)$ and the pure vortex-driven mechanism expected in the second non-linear regime $\left(N_{2}\right)$.

Finally, in the second non-linear regime $\left(N_{2}, A / U_{b}=8.92 \%\right.$ and $\left.35.7 \%\right)$, the space-time correlation is dominated by a convective oscillation. A damping zone appears close to the upstream edge because of the vortex generation at time $0<t<T / 2$. This vortex is then convected downstream at a speed slower than $W / T$, here approximately $u_{\Gamma} \approx W / 2 T$. In the second half of the period $(T / 2<t<T)$, a strong production zone exists. The convection speed is now higher than $W / T$, i.e. $u_{\Gamma} \approx 3 W / 2 T$, because of the vortex deviation, as observed in figure 12 . This deviation also reduces the vortex-corner interaction, leading to a maximum constructive coupling located closer to the middle of the cavity $x=0$, whereas for the two other regimes it was located at the downstream edge $x \approx W / 2$. It can be also noticed that for large amplitudes $\left(A / U_{b}=35.7 \%\right)$, a second strong damping zone is observed at a latter time $t \approx T / 2$. It corresponds to the second vortex generated during the blow-off phase, already observed in figure 12. Note that the production zone associated with this second vortex is weak, but can be seen at time $0<t<T / 2$ because of the $x-t$ plane periodicity. 


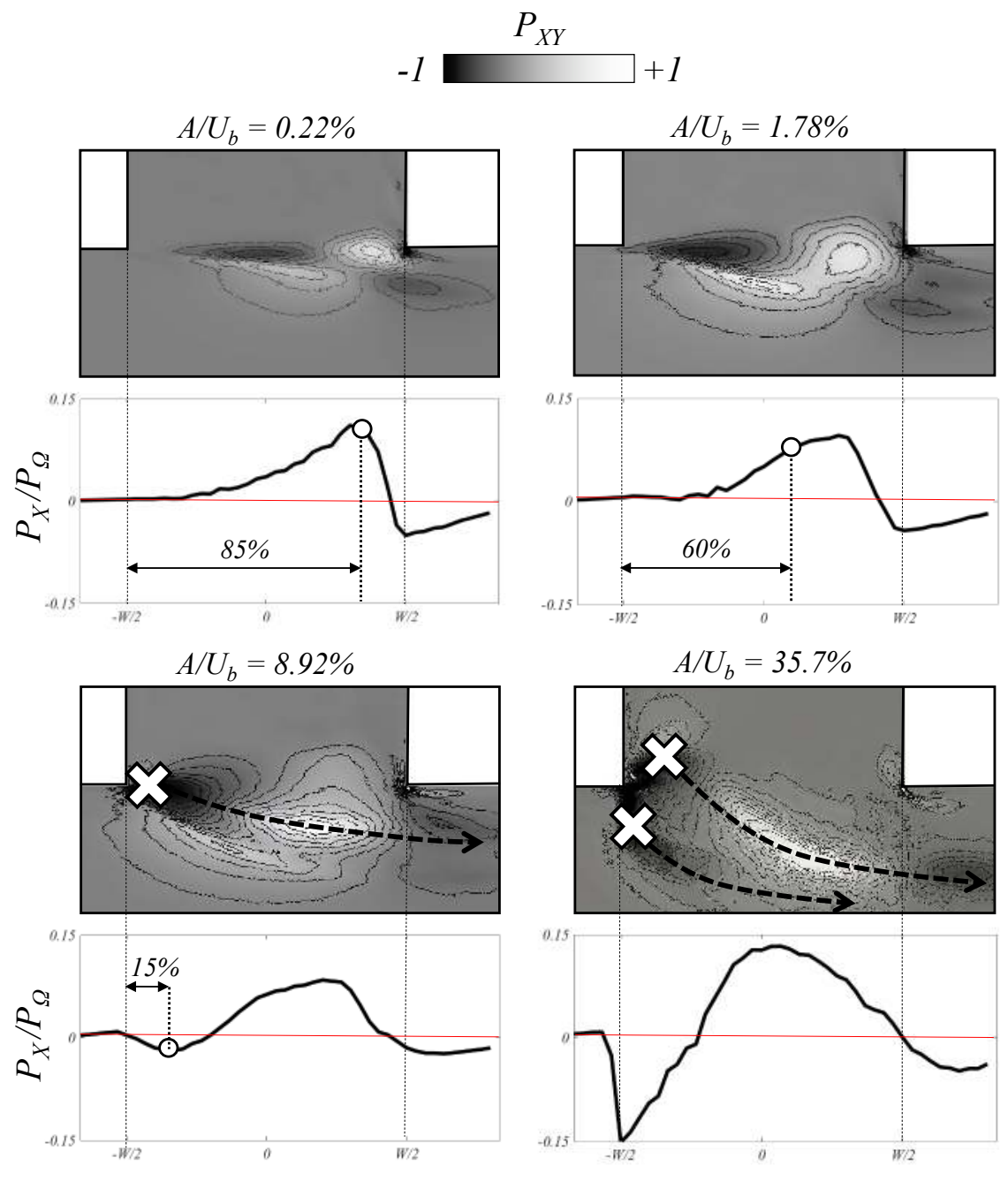

Fig. 14 Local time-averaged power source $P_{X Y}$ at $750 \mathrm{~Hz}$ for several amplitudes $A / U_{b}$. Results are adimensionalized, white zones corresponding to a positive power, and black to negative values. The arrows display the vortex deviation. The normalized y-integrated power source $P_{X} / P_{\Omega}$ is also shown for each amplitude.

\section{Local analysis of the vortex-sound coupling}

In complement with the space-time analysis where fluctuations along the $y$-axis have been averaged, a local analysis of the vortex-sound coupling is performed in the $x-y$ plane. To do so, the local time-averaged power sources $P_{X Y}$ and $P_{X}$ (equations (7)-(8)) are computed by LES. $P_{X}(x)$ is computed by the integration of $P_{X Y}$ along the y-coordinate over a sliding band $x \pm \Delta x / 2$, where $\Delta x=1 \mathrm{~mm}$. The post-processing to obtain $P_{X Y}$ is similar to the ones for $P_{X T}$, detailed in Section V.B. Typical results for a simplified flapping shear layer and vortex shedding are given in figure 5. LES results are displayed in figure 14 for various wave amplitudes. White zones correspond to production of sound, and dark ones to damping zones. In addition, the y-integrated power source $P_{Y}$ is provided in figure 14 for each amplitude, normalized by the time-averaged total power $\left\langle P_{\Omega}\right\rangle_{T}$.

First, the amplitude $A / U_{b}=0.22 \%$ corresponds to the linear regime ( $L$ in figure 11). The $P_{X Y}$ map reveals two upstream zones (damping and production) which lie on both side (top and bottom) of the mean shear layer position. As reported in the scenario $a$ ) of figure 2, such a response is due to the flapping motion, also identified in figure 13 . This flapping motion takes place over $70 \%$ of the cavity width. In this region, $P_{X}$ varies exponentially, as for the flapping phenomenological model drawn in figure 4 . In particular, no damping zone due to a vortex creation is obtained at the upstream edge. However, compared with the flapping model of figure 5, a weak roll-up of the shear layer occurs further downstream, which enhances the vortical flow in the second half of the cavity. The downstream corner also exhibits a 
small damping region due to the shear layer interacting with the corner. Overall, $P_{X}$ is typical of a flapping motion. The location of the maximum of unsteadiness obtained by the modal decomposition at $750 \mathrm{~Hz}$ (figure 8) is also shown in $P_{X}(\circ)$ : this location corresponds to the largest constructive coupling in the cavity. As a conclusion, the linear regime mainly exhibits a flapping motion, and is restricted for this configuration to very low amplitudes $A / U_{b}<0.4 \%$.

At a moderate amplitude $A / U_{b}=1.78 \%$, the describing function reveals that the situation corresponds to the first non-linear regime $\left(N_{1}\right)$, exhibiting both a flapping motion and an intense roll-up. Compared to the linear regime, this roll-up takes place further upstream, in the present case at $60 \%$ of the cavity width. Indeed, before this location (o), $P_{X}$ evolves similarly than for the linear regime. The $P_{X Y}$ shows a typical damping/production zone which lies on both side (top/bottom) of the mean shear layer. However, further downstream, the roll-up leads to a large production zone followed by a weak damping zone. This spatial distribution of the damping/production zones along the x-axis, corresponding to the convection direction, is typical of a discrete vortex model, as shown in figure 5 . $P_{X}$ indicates that the sound production in the production zone after the roll-up is nearly constant, i.e., $P_{X} / P_{\Omega} \approx 0.08$ (blue dashed line). Again, the downstream edge exhibits a damping zone due to the vortex-corner interaction. As expected from the modal decomposition in figure 8 and the space-time analysis in figure 13 , this situation corresponds to a combination of an upstream flapping motion followed by a significant downstream roll-up. Consequently, in such a case, neither the flapping model or the discrete vortex model is representative of the shear layer oscillations.

At larger amplitudes, the second non-linear regime $\left(N_{2}\right)$ appears. The case at $A / U_{b}=8.92 \%$ is typical of the transition between $N_{1}$ and $N_{2}$, while $A / U_{b}=35.7 \%$ corresponds to the pure $N_{2}$ regime. In the temporal snapshots, an intense discrete vortex is shed at the upstream corner of the T-junction. Over the whole cavity, the damping and production zones shown by $P_{X Y}$ are now aligned along the direction of the vortex convection, highlighted by the red arrows. The vortex generated at the upstream edge (marked by $\times$ in figure 14) is convected by the mean flow at a speed close to $0.4 U_{b}$ so that the convection time from one corner to the other is approximately $\tau=W_{X} /\left(0.4 U_{b}\right) \approx 1.34 \mathrm{~ms}$. This convection time is locked with the acoustic period $T_{a c}=1 / f=1.33 \mathrm{~ms}$. For $A / U_{b}=35.7 \%$, a second vortex is created during the blow-off $\left(v^{\prime}<0\right)$, which enhances the upstream damping zone, as observed by the large negative value of $P_{X}$. A deviation of the vortex away from the side-tube can be observed for both $A / U_{b}=8.92 \%$ and $35.7 \%$ in the second half of the cavity $(0<x<W / 2)$, due to the intense acoustic velocity during the blow-off phase. It yields a reduction of the vortex-corner interaction at the downstream edge $\left(P_{X}\right.$ is almost null at $\left.x=W / 2\right)$. For the largest amplitude, this deviation also diminishes the production term, since the transverse acoustic velocity decreases drastically away from the side-branch (figure 9p. In this regime, the vortex-discrete model holds and the vortex-corner interaction can be ignored.

This complex view of the shear layer behavior changing with the forcing amplitude $A / U_{b}$ lies between the two classical models (discrete vortex and continuously oscillating shear layer). In particular, for moderate acoustic levels, both scenarios co-exist with an upstream flapping of the shear layer, followed by a roll-up and a vortex creation. Such a study is in agreement with recent experimental observations performed by Ma et al. [31] in a similar case, where both flapping and vortex shedding were found. This section has described qualitatively the differences in the shear layer behaviors depending on the wave amplitude, pointing out key physical phenomena (flapping, vortex generation, vortex deviation, interaction at the downstream corner etc.).

\section{The non-linear hydrodynamic feedback loop}

The spatio-temporal analysis has revealed that when a larger acoustic forcing is applied, larger vortex structures are shed, which increases as well the apparent thickness of the unsteady shear layer. This thickening of the unsteady shear layer can be linked to the evolution of the underlying baseline flow $\vec{u}_{0}$, which is associated with the non-linearities present in the system. To unravel these non-linear effects, a convenient approach is to split the non-linear problem into two coupled systems, i.e. a mean flow equation together with a linear perturbation equation around this mean flow. This approach, known as "self-consistent", was proposed by Mantic-Lugo and Gallaire [44] to tackle non-linear stability problems in fluid mechanics. Recently, Boujo et al. [42] have conducted a similar study on the present configuration, where the mean flows were extracted from LES simulations at several amplitudes $A / U_{b}$ instead of solving the baseline flow equation. They have shown a good agreement in the describing function between the full non-linear LES and the linearized approach, revealing that the non-linearities of the current problem are hidden in the baseline flow evolution with the wave amplitude $A / U_{b}$. This approach is performed here as an alternative to identify and investigate the very source of non-linearity leading to the saturation of the shear layer in the $N_{1}$ and $N_{2}$ regimes.

The first system corresponds to the linear evolution of the fluctuation $\overrightarrow{\hat{u}}$, which is computed from the baseline flow $\vec{u}_{0}$ through a linear equation. Assuming that only the acoustic forcing $A \hat{f}$ is affecting the mode at $750 \mathrm{~Hz}$, meaning that 


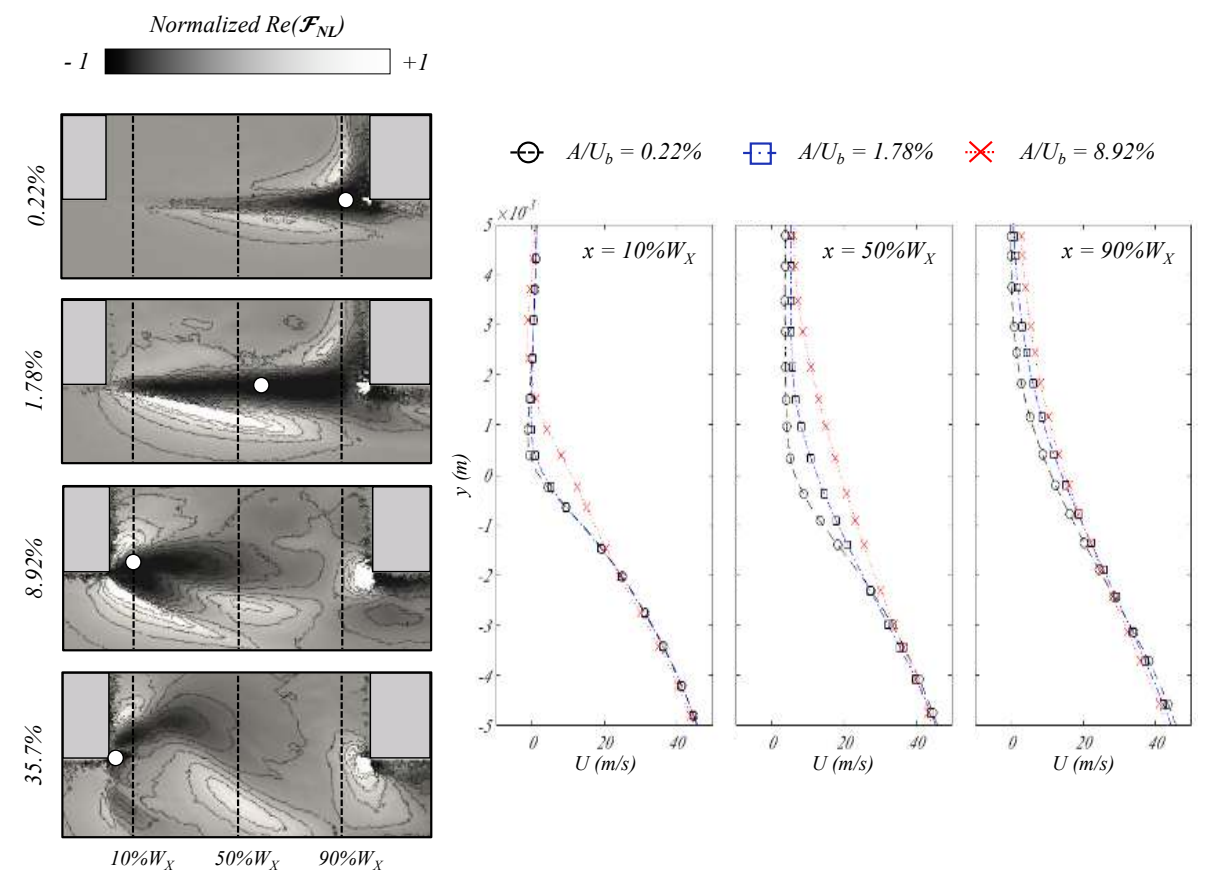

Fig. 15 The real part of the hydrodynamic feedback term $\mathcal{F}_{N L}$ (equation 18 ) for several wave amplitudes $A / U_{b}$. The minimal value of $\mathcal{F}_{N L}$ is marked by $\circ$. The axial velocity profile at three positions in the cavity are also given (right), highlighting the thickening of the steady shear layer.

harmonics can be neglected, this equation reduces to

$$
j \omega_{1} \overrightarrow{\hat{u}}+L\left(\vec{u}_{0}\right) \overrightarrow{\hat{u}}=A \hat{f},
$$

where $\hat{f}$ is a normalized forcing velocity at the tube tip, $\omega_{1}$ is the complex angular frequency of the mode accounting for the frequency of oscillations at $750 \mathrm{~Hz}$ and its growth rate, and $L\left(\vec{u}_{0}\right) \overrightarrow{\hat{u}}=\left(\vec{u}_{0} \cdot \vec{\nabla}\right) \overrightarrow{\hat{u}}+(\overrightarrow{\hat{u}} \cdot \vec{\nabla}) \vec{u}_{0}+\frac{1}{\rho} \vec{\nabla} \hat{p}-\nu \vec{\nabla}^{2} \overrightarrow{\hat{u}}$ is the Navier-Stokes operator linearized around the mean flow. In the linear regime $(L)$, this equation is sufficient to capture the exponential growth of the mode at $750 \mathrm{~Hz}$ given the mean flow $\vec{u}_{0}$ as input. Since this equation is purely linear, the non-linear mechanism is necessarily included in the other coupled equations. Boujo et al. [42] have shown that in the present case, the non-linearity is contained in the evolution of the baseline flow, and not because of higher-harmonics modes. It therefore corresponds to a non-linear feedback loop between the first harmonic and the baseline flow. Assuming a negligible effect of the coherent high-order harmonics and incoherent turbulent fluctuations, the evolution of the baseline flow $\vec{u}_{0}$ reads

$$
N\left(\vec{u}_{0}\right)=-\mathcal{F}_{N L} \text { where } \mathcal{F}_{N L}=\overrightarrow{\hat{u}} \cdot \vec{\nabla} \hat{u}^{*}+\overrightarrow{\hat{u}}^{*} \cdot \vec{\nabla} \hat{u}
$$

where $N\left(\vec{u}_{0}\right)=\left(\vec{u}_{0} \cdot \vec{\nabla}\right) \vec{u}_{0}+\frac{1}{\rho_{0}} \vec{\nabla} p_{0}-v \vec{\nabla}^{2} \vec{u}_{0}$ is the incompressible Navier-Stokes operator, and * denotes the complex transpose. $\mathcal{F}_{N L}$ is therefore a non-linear term acting as a feedback loop propagating back the information of the coherent fluctuations $\hat{u}$ on the baseline flow $\vec{u}_{0}$. In the linear regime, this non-linear term does not affect the mean flow which is therefore the steady solution of $N\left(\vec{u}_{0}\right)=0$ : for all amplitudes $A / U_{b}$ in the linear regime, the mean flow $\vec{u}_{0}$ remains the same. As predicted by equation (17), the velocity fluctuation evolves linearly with the amplitude $A$.

However, in the non-linear regimes $N_{1}$ and $N_{2}$, the feedback $\mathcal{F}_{N L}$ becomes non-negligible and affects the baseline flow, which therefore evolves non-linearly with the wave amplitude $\vec{u}_{0}=\vec{u}_{0}\left(A / U_{b}\right)$. Since the mean flow is changing, the solution of equation (17) is no more linear with the amplitude $A / U_{b}$. Note that Boujo et al. [42] have shown that solving the linear equation (17) using the mean flow $\vec{u}_{0}\left(A / U_{b}\right)$ extracted from LES simulations allows a good approximation of the describing function displayed in figure 11. In this view, all the non-linearities are contained in the feedback term $\mathcal{F}_{N L}$, which therefore has to be investigated in details to understand the non-linear processes controlling $N_{1}$ and $N_{2}$. Based on equation (18), this feedback term can be computed for several acoustic levels $A / U_{b}$ using the modal 


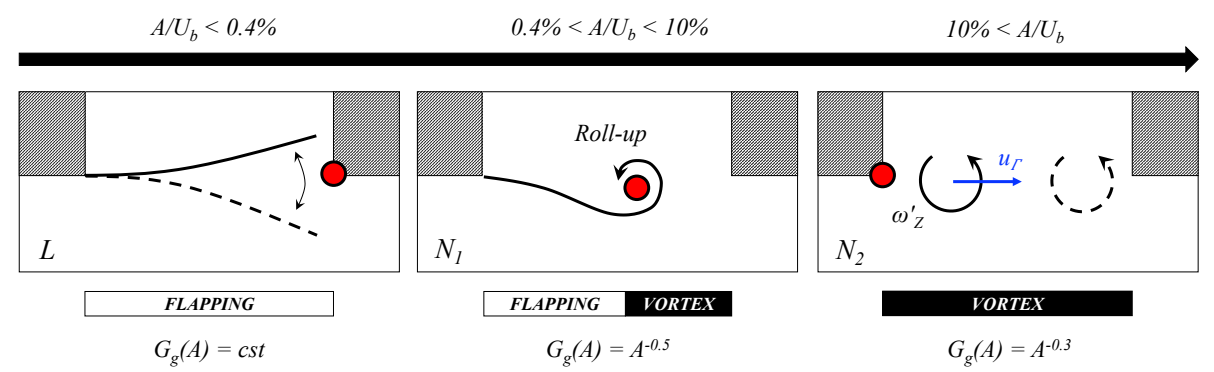

Fig. 16 Conclusion on the three regimes $\left(L, N_{1}\right.$, and $\left.N_{2}\right)$ vs. the wave amplitude $A / U_{b}$ observed in the present T-junction. The shear layer behavior is represented for each case, and marked as flapping or vortex shedding. The evolution of the gain of the describing function $G_{g}$ with the wave amplitude is also reported. The location where $u^{\prime}$ is maximum is displayed by $\circ$, which coincides with the minimal value of $\mathcal{F}_{N L}$.

decomposition at $750 \mathrm{~Hz}$ shown in figure 8 . Results are displayed in figure 15 (left). Since in the linear regime $(L)$ all modal decompositions $\hat{u}$ are similar, it results that all feedback term $\mathcal{F}_{N L}$ are also similar, therefore only $A / U_{b}=0.22 \%$ is shown (top). Interestingly, the predominance of the downstream corner in the linear regime $(L)$ is retrieved. At higher amplitudes, the modal decomposition and the feedback term are changing with the wave amplitude $A / U_{b}$. In contrast with the linear regime where $\mathcal{F}_{N L}$ is concentrated at the downstream edge, the feedback term is located closer to the upstream edge in the second non-linear regime $\left(N_{2}\right)$. In the first non-linear regime $N_{1}$, corresponding to a combination of a flapping and roll-up motions, $\mathcal{F}_{N L}$ is acting over the whole cavity. Consequently, it shows that $\mathcal{F}_{N L}$ is a good indicator on which regime $\left(L, N_{1}\right.$ or $\left.N_{2}\right)$ is at stake, and can be used on the modal decomposition of a self-excited limit cycle if needed.

Since the non-linear hydrodynamic feedback loop is directly acting on the mean flow field, its effect can be noticed on the baseline flow. Figure 15 (right) shows the mean axial velocity profiles for three wave amplitudes, and three spatial locations $(10 \%, 50 \%$ and $90 \%$ of the cavity width). As depicted by the spatial location of the feedback term, the mean shear layer is thickened first at the downstream edge, and becomes thicker further upstream only for larger wave amplitudes because of the build up of the Reynold stresses. It suggests that the hydrodynamic feedback is intense at the location corresponding to the roll-up/vortex creation, starting from the downstream edge at low wave amplitudes and moving further upstream at larger acoustic levels. This is quantified by the minimal value of the feedback term, marked by $\circ$ in figure 15 , which corresponds to $90 \%$ of the cavity width at $A / U_{b}=0.22 \%, 60 \%$ at $A / U_{b}=1.78 \%$, and $10 \%$ for $A / U_{b}=8.92 \%$. Note that these locations coincide with the maximal values of $\hat{u}$, as reported in figure 8 .

Thus, it has been shown that the non-linearities of the system are contained in the non-linear hydrodynamic feedback loop. This feedback term depends non-linearly on the fluctuation $\hat{u}$, which implies that it is maximum at the location of the roll-up or vortex shedding, since these mechanisms induce a large perturbation $\hat{u}$. In the linear regime, such a roll-up can only appear at the downstream edge because of the shear layer interacting with the the corner. The feedback term is therefore located close to the downstream edge, leading to a thickening of the shear layer at this location. In turns, it modifies the response of the shear layer to acoustic waves $\hat{u}$, yielding a build up of Reynolds stresses and non-linearities further upstream. This backward propagation of non-linearities allows an early roll-up of the shear layer, thereby promoting a more upstream generation of vortical structures and its associated shear layer thickening: this regime corresponds to $N_{1}$. Once the non-linear hydrodynamic feedback loop induces vortical structures at the upstream corner, the back propagation stops. Non-linearities accumulate at the upstream corner, yielding a vortex shedding attached at the upstream edge: this is the second non-linear regime $\mathrm{N}_{2}$. Consequently, it appears that the backward propagation of the non-linear feedback term is the key feature making the transition from the linear to the non-linear regimes possible.

\section{Conclusion}

Many investigations have studied the fundamental vortex-noise coupling in academic T-junctions. They usually focus on deriving low-order models to predict the amplitude of oscillations in the limit cycle. Yet numerous questions are still opened today, such as the identification of the nonlinear mechanisms controlling the saturation of these oscillations, and how the flow evolves from a linear to a non-linear behavior. The present paper intends to analyse such nonlinearities in a T-junction to clarify the role of the upstream and downstream corners and their associated phenomena in the vortex-noise 
interaction. This study relies on forced Large Eddy Simulations (LES) applied to an academic T-junction exhibiting a strong instability at $750 \mathrm{~Hz}$ due to the interaction between the vortical flow and the quarter-wave mode of the side branch. This interaction can be extracted by computing the classical vortex-noise source term in the form of a describing function. Such a function relates the unsteady Coriolis force to the acoustic velocity through a gain and a phase, both depending on the frequency and amplitude of the injected acoustic wave. Surprisingly, three different regimes can be identified: (L) a linear regime characterized by a constant gain at low acoustic levels, $\left(N_{1}\right)$ a first non-linear regime corresponding to moderate wave amplitudes, and a second non-linear regime $\left(N_{2}\right)$ for high amplitudes. An in-depth look at the flow using a modal decomposition and the computation of the local power source map unveils that the flapping motion of the vortex sheet observed at low amplitudes, leaves the place to a vortex shedding at high amplitudes. At moderate amplitudes corresponding to $N_{1}$, both the flapping motion and an intense roll-up of the shear layer co-exist. The modification of the unsteady behavior of the shear layer can be explained by the hydrodynamic feedback loop acting on the baseline flow, associated with the non-linearities of the system. For all regimes, it is strongly correlated with the roll-up and vortex generation. Thus, the hydrodynamic feedback is dominant at the downstream edge in the linear regime $(L)$, whereas it is significant at the upstream corner for the second non-linear regime $\left(N_{2}\right)$. In $N_{1}$, this feedback loop is spreading over the whole cavity. It implies that the thickening of the mean shear layer occurs first at the downstream edge, and then propagates backward, closer to the upstream edge, along the build-up of the Reynold stresses due to the further upstream roll-up of the shear layer at larger wave amplitudes. This study therefore provides a global view of the mechanisms controlling the three different behaviors of the shear layer instability, and are summarized in figure 16. Such analysis examplifies that simple "discrete vortex" or "continuously oscillating" models for the shear layer response to acoustic waves are limited. As illustrated in figure 16, a more realistic view lies between the two classical theoretical models, as suggested by Ma et al. [31] and Morris [32].

\section{A. Linearized momentum balance equation}

It has been shown that the unsteady Coriolis force $f_{c}^{\prime}$ is acting as a dipolar source term in the Helmholtz equation (3), and is therefore used to define the describing function $\mathcal{F}_{g}$. Nevertheless, classical approximations are often made for the sake of simplicity, for instance $f_{c}^{\prime} \approx-\rho_{0} \omega_{Z}^{\prime} u_{0}$. Similarly, because $f_{c}^{\prime}$ is difficult to characterize experimentally, Graf and Ziada [15] measured the describing function based on the pressure jump across the shear layer. These several approximations can be questioned, especially at large acoustic levels.

These quantities are related to the linearized momentum balance equation. When projected on the $y$-direction, and integrated over the subdomain $\Omega$, equation $(2)$ becomes

$$
\underbrace{\int_{\Omega} \rho_{0} \frac{\partial v^{\prime}}{\partial t} d \Omega+\rho_{0}\left[B^{\prime}\right]}_{\text {LHS } 1}=\underbrace{\int_{\Omega} \vec{f}_{c}^{\prime} \cdot \vec{y} d \Omega}_{\text {RHS } 1}
$$

where $\left[B^{\prime}\right]$ is the enthalpy jump accross the shear layer in the $y$-direction, usually approximated by the unsteady pressure at low Mach number $B^{\prime} \approx p^{\prime} / \rho_{0}$. Consequently, the enthalpy or pressure jumps are a good approximation of the shear layer response only if the inertia term $\rho_{0} \frac{\partial v^{\prime}}{\partial t}$ is negligible: in such a case, the LHS term reduces to LHS $2=\rho_{0}\left[B^{\prime}\right]$. In complement, the validity of the classical approximation of the Coriolis force $f_{c}^{\prime} \approx-\rho_{0} \omega_{Z}^{\prime} u_{0}$ is assessed by approximating the RHS term by RHS $2=-\int_{\Omega} \rho_{0} \omega_{Z}^{\prime} u_{0} d \Omega$.

These several quantities (LHS 1, LHS 2, RHS 1, and RHS 2) are evaluated by LES in case $C$ at several amplitudes. Their computation is made through $2 \mathrm{D}$ cuts extracted every $\Delta t=100 \mu \mathrm{s}$, after a filtering around $750 \mathrm{~Hz}$. The integration is done on the subdomain $\Omega_{m v}$, which encapsulates the shear layer while ensuring the compactness assumption.

Results are provided in figure 17 . They reveal that at low amplitudes $\left(A / U_{b}=0.045 \%\right)$, no difference can be noticed between the several approximations, and a good balance is obtained between the LHS and RHS terms. At moderate amplitudes $\left(A / U_{b}=0.13 \%\right.$ and $\left.0.45 \%\right)$, the inertia term is still weak so that LHS $1 \approx$ LHS 2 , meaning the enthalpy jump is still a relevant quantity to evaluate the response of the shear layer to acoustic waves. However, the RHS 2 ( $\square$ in figure 17p approximation deteriorates: the complete unsteady Coriolis force $f_{c}^{\prime}$ should be used. Finally, at larger amplitudes both LHS $2(\times)$ and RHS $2(\square)$ are no more valid. The correct balance equation is recover only with the complete expression LHS 1 ( $\circ$ ) and RHS $1(+)$. Consequently, in this paper the complete Coriolis force $f_{c}^{\prime}$ is used for the describing function which ensures a correct description of the shear layer response to acoustic waves, even in the non-linear regime. 

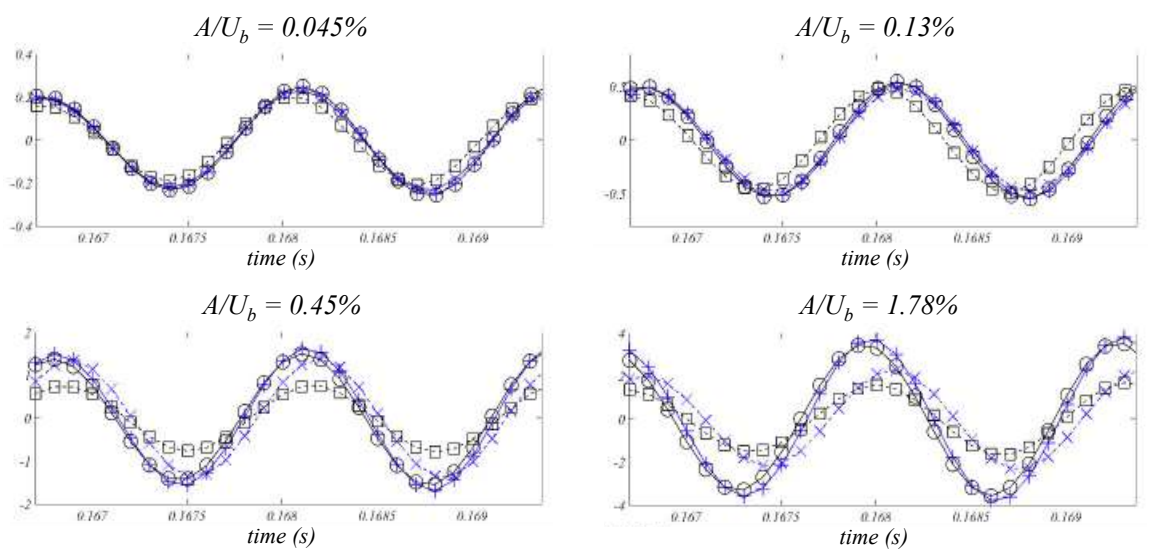

$\longrightarrow \operatorname{LHS} 1-\Varangle-\operatorname{LHS} 2$

$\odot$ RHS $1-\mathrm{E}-\mathrm{RHS} 2$

Fig. 17 The LHS and RHS terms of the momentum balance equation are computed with several approximations for various acoustic levels $A / U_{b}$.

\section{References}

[1] Chu, B.-T., and Kovasznay, L., "Non-linear interactions in a viscous heat-conducting compressible gas," J. Fluid Mech. , Vol. 3 , No. 5, 1958, pp. 494-514.

[2] Maung, P., Howe, M., and McKinley, G., "Experimental investigation of the damping of structural vibrations by vorticity production,” J. Sound Vib. , Vol. 220, No. 2, 1999, pp. 297-312.

[3] Rienstra, S., and Hirschberg, A., An introduction to acoustics, 2004.

[4] Huerre, P., and Monkevitz, P., "Local and global instabilities in spatially developped flows," Annual review of fluide mechanics, Vol. 22, 1990, pp. 473-537.

[5] Powell, A., "Theory of vortex sound,” J. Acous. Soc. Am. , Vol. 36, No. 1, 1964, pp. 177-195.

[6] Rockwell, D., “Oscillation of impinging shear layer,” AIAA Journal, Vol. 21, 1983, pp. 645-664.

[7] Howe, M., "Edge, cavity and aperture tones at very low Mach numbers," J. Fluid Mech., Vol. 330, 1997, pp. 61-84.

[8] Rowley, C., and Williams, E., "Dynamics and control of High-Reynolds-Number flow over open cavities," Annu. Rev. fluid Mech., Vol. 38, No. 251-276, 2006.

[9] Rockwell, D., and Naudascher, E., "Review: self-sustaining oscillations of flow past cavities," Trans. ASME. J. Fluids Eng. , Vol. 100, 1978, pp. 152-165.

[10] Dequand, S., Hulshoff, S., and Hirschberg, A., "Self-sustained oscillations in a closed side branch system," J. Sound Vib. , Vol. 265, 2003, pp. 359-386.

[11] Tonon, D., Willems, J., and Hirschberg, A., "Self-sustained oscillations in pipe systems with multiple deep side branches: prediction and reduction by detuning," J. Sound Vib. , Vol. 330, 2011, pp. 5894-5912.

[12] Pollack, M., "Flow-induced tones in side-branch pipe resonators," J. Acoust. Soc. Am., Vol. 67, No. 4, 1980 , pp. $1153-1156$.

[13] Ziada, S., and Shine, S., "Strouhal numbers of flow-excited acoustic resonance of closed side branches," J. Fluids Struct., Vol. 13, No. 1, 1999, pp. 127-142.

[14] Geveci, M., Oshkai, P., Rockwell, D., Lin, J.-C., and Pollack, M., "Imaging of the self-excited oscillation of flow past a cavity during generation of a flow tone,” J. Fluids Struct., Vol. 18, 2003, pp. 665-694.

[15] Graf, H. R., and Ziada, S., “Excitation source of a side-branch shear layer,” J. Sound Vib. , Vol. 329, 2010, pp. $2825-2842$. 
[16] Karlsson, M., and Abom, M., "Aeroacoustics of T-junction: An experimental investigation,” J. Sound Vib. , Vol. 329, 2010, pp. 1793-1808.

[17] Comte, P., Daude, F., and Mary, I., "Simulation of the reduction of unsteadiness in a passively controlled transonic cavity flow," J. Fluids Struct., Vol. 24, 2008, pp. 1252-1261.

[18] Nakiboglu, G., Manders, H., and Hirschberg, A., "Aeroacoustic power generated by a compact axysymmetric cavity: prediction of self-sustained oscillation and influence of the depth," J. Fluid Mech., Vol. 703, 2012, pp. 163-191.

[19] Gikadi, J., Foller, S., and Sattelmayer, T., "Impact of turbulence on the prediction of linear aeroacoustic interactions: acoustic response of a turbulent shear layer," J. Sound Vib. , Vol. 333, 2014, pp. 6548-6559.

[20] Howe, M. S., "The generation of sound by aerodynamic sources in an homogeneous steady flow," J. Fluid Mech. , Vol. 67, No. 3, 1975, pp. 597-610.

[21] Myers, M., "An exact energy corollary for homentropic flow," J. Sound Vib. , Vol. 109, 1986, pp. $277-284$.

[22] Brear, M., Nicoud, F., Talei, M., Giauque, A., and Hawkes, E., "Disturbance energy transport and sound production in gaseous combustion," J. Fluid Mech. , Vol. 707, 2012, pp. 53-73.

[23] Elder, S., "Self-excited depth-mode resonance for a wall-pounted cavity in turbulent flow," J. Acoust. Soc. Am., Vol. 64, 1978, pp. $877-890$.

[24] Howe, M., "The influence of mean shear on unsteady aperture flow, with application to acoustical diffraction and self-sustained cavity oscillations," J. Fluid Mech., Vol. 109, 1981, pp. 125-146.

[25] Chatelier, L., Laumonier, J., and Gervais, Y., "Theoretical and experimental investigations of low Mach number turbulent cavity flows," Experiments in Fluids, Vol. 36, 2004, pp. 728-740.

[26] Nelson, P., Halliwell, N., and Doak, P., "Fluid dynamics of a flow excited resonance. Part II: flow acoustic interaction," J. Sound Vib. , Vol. 91, 1983, pp. 375-402.

[27] Bruggeman, J., Hirschberg, A., Dongen, M. V., and Wijnands, A., "Self-sustained aero-acoustic pulsations in gas transport systems: experimental study of the influence of closed side branches," J. Sound Vib. , Vol. 150, No. 3, 1991, pp. 371-393.

[28] Mast, T., and Pierce, A., "Describing-function theory for flow excited resonators," J. Acoust. Soc. Am., Vol. 97, 1995, pp. $163-172$.

[29] Kook, H., and Mongeau, L., "Analysis of the periodic pressure fluctuations induced by flow over a cavity," J. Sound Vib. , Vol. 251, 1997, pp. 823-846.

[30] Meissner, M., "the response of a Helmholtz resonator to external excitation. Part II: flow-induced resonance," Arch. Acoust., Vol. 30, 2005, pp. 57-71.

[31] Ma, R., Slaboch, P., and Morris, S., "Fluid mechanics of the flow-excited Helmholtz resnator," J. Fluid Mech. , Vol. 623, 2009, pp. 1-26.

[32] Morris, S., "Shear-layer instabilities: Particle Image Velocimetry measurements and implications for acoustics," Annu. Rev. fluid Mech., Vol. 43, 2011, pp. 529-550.

[33] Dai, X., Jing, X., and Sun, X., "Flow-excited acoustic resonance of a Helmholtz resonator: Discrete vortex model compared to experiments," Phys. Fluids, Vol. 27, 2015, p. 057102.

[34] Rayleigh, L., The Theory of Sound, Mac Millan (reprinted by Dover, New York, 1945), 1894.

[35] Michalke, A., “On spatially growing disturbances in an inviscid shear layer,” J. Fluid Mech. , Vol. 23, No. 3, 1965 , pp. 521-544.

[36] Krylov, N., and Bogoliubov, N., Introduction to Nonlinear Mechanics, Princeton University Press, 1943.

[37] Lighthill, M. J., “On Sound Generated Aerodynamically. I. General Theory,” Proc. R. Soc. Lond. A , Mathematical and Physical Sciences, Vol. 211, No. 1107, 1952, pp. 564-587.

[] Poinsot, T., and Lele, S., "Boundary conditions for direct simulations of compressible viscous flows," J. Comput. Phys. , Vol. 101, No. 1, 1992, pp. 104-129. https://doi.org/10.1016/0021-9991(92)90046-2 
[39] Nicoud, F., Baya-Toda, H., Cabrit, O., Bose, S., and Lee, J., "Using singular values to build a subgrid-scale model for Large Eddy Simulations," Physics of fluids, Vol. 23, No. 085106, 2011, pp. 1-12.

[40] Colin, O., and Rudgyard, M., "Development of high-order Taylor-Galerkin schemes for unsteady calculations," J. Comput. Phys., Vol. 162, No. 2, 2000, pp. 338-371.

[41] Selle, L., Lartigue, G., Poinsot, T., Koch, R., Schildmacher, K.-U., Krebs, W., Prade, B., Kaufmann, P., and Veynante, D., "Compressible Large-Eddy Simulation of turbulent combustion in complex geometry on unstructured meshes," Combust. Flame, Vol. 137, No. 4, 2004, pp. 489-505.

[42] Boujo, E., Bauerheim, M., and Noiray, N., "Saturation of a turbulent mixing layer over a cavity: response to harmonic forcing around mean flows," J. Fluid Mech., Vol. 853, 2018, pp. 386-418.

[43] Nicoud, F., Benoit, L., Sensiau, C., and Poinsot, T., "Acoustic modes in combustors with complex impedances and multidimensional active flames," AIAA Journal , Vol. 45, 2007, pp. 426-441.

[44] Mantic-Lugo, V., and Gallaire, F., "Self-consistent model for the saturation mechanism of the response to harmonic forcing in the backward-facing step flow," J. Fluid Mech., Vol. 793, 2016, pp. 777-797. 